\title{
Angewandte
}

Supporting Information

(C) Wiley-VCH 2011

69451 Weinheim, Germany

\section{A Boronium Ion with Exceptional Electrophilicity**}

Aleksandrs Prokofjevs, Jeff W. Kampf, and Edwin Vedejs*

anie_201005663_sm_miscellaneous_information.pdf 


\section{Table of Contents}

N-(Borabicyclo[3.3.1]nonan-9-yl)-1,1,1-trifluoro-N-

(trifluoromethanesulfonyl)methanesulfonamide (5a) 3

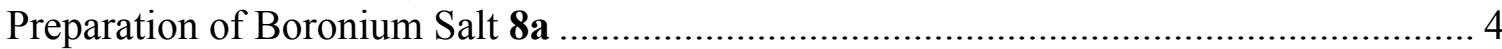

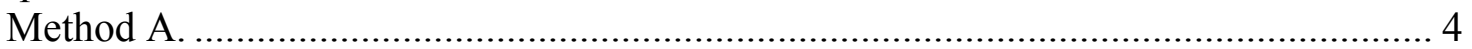

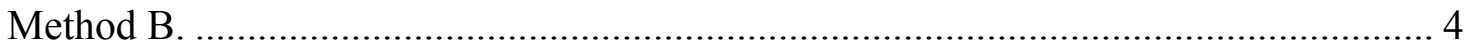

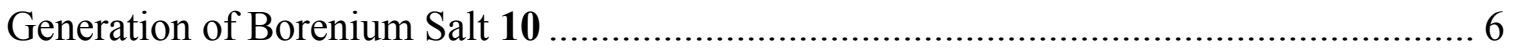

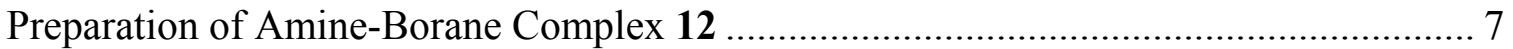

Generation of Borenium Salt 13 and Boronium Salt 14 f............................................ 8

Representative Procedure for Indole and Pyrrole Borylation ........................................ 10

3-(9-Borabicyclo[3.3.1]nonan-9-yl)-1-methyl-1H-indole (16a) ................................. 10

3-(9-Borabicyclo[3.3.1]nonan-9-yl)-1,2,5-trimethyl-1H-pyrrole (16b) ........................ 11

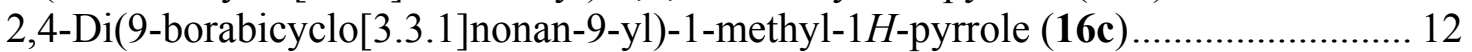

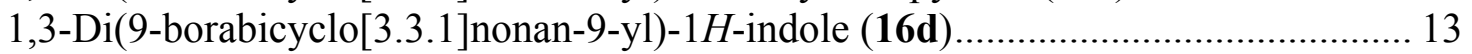

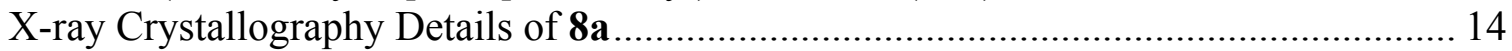

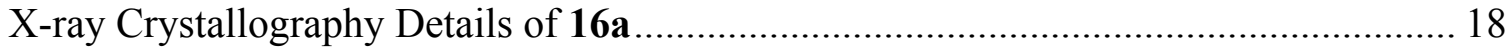

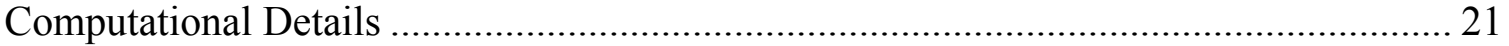

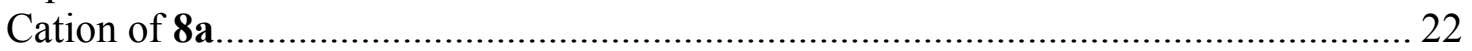

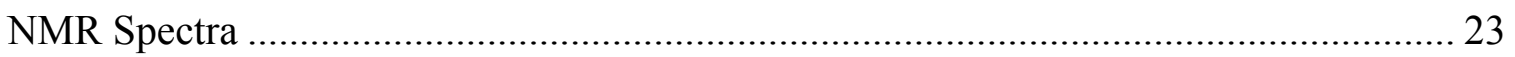


All reactions were performed at room temperature (unless otherwise stated), under an atmosphere of dry nitrogen, either in a glovebox, or using standard Schlenk techniques. Nuclear magnetic resonance experiments were performed on Varian Inova 500 and Inova 400 spectrometers at the following frequencies: ${ }^{1} \mathrm{H} 500 \mathrm{MHz}$ or $400 \mathrm{MHz} ;{ }^{13} \mathrm{C}\left\{{ }^{1} \mathrm{H}\right\}$ $101 \mathrm{MHz} ;{ }^{11} \mathrm{~B}$ and ${ }^{11} \mathrm{~B}\left\{{ }^{1} \mathrm{H}\right\} 128 \mathrm{MHz} ;{ }^{19} \mathrm{~F} 377 \mathrm{MHz}$. All spectra were recorded in $\mathrm{CD}_{2} \mathrm{Cl}_{2}$ and referenced to the ${ }^{1} \mathrm{H}$ signal of internal $\mathrm{Me}_{4} \mathrm{Si}$ according to IUPAC recommendations, ${ }^{33}$ using a $\Xi$ of 25.145020 for $\mathrm{Me}_{4} \mathrm{Si}\left({ }^{13} \mathrm{C}\right)$, a $\Xi$ of 32.083974 for $\mathrm{BF}_{3}-\mathrm{OEt}_{2}\left({ }^{11} \mathrm{~B}\right)$, and a $\Xi$ of 94.094011 for $\mathrm{CCl}_{3} \mathrm{~F}\left({ }^{19} \mathrm{~F}\right)$. IR spectra were acquired in $\mathrm{CCl}_{4}$ or $\mathrm{CD}_{2} \mathrm{Cl}_{2}$ solutions using a $\mathrm{CaF}_{2}$ cell. UV spectra were acquired using a Shimadzu UV-1601 spectrophotometer. Toluene and $\mathrm{NEt}_{3}$ were distilled over $\mathrm{CaH}_{2}, \mathrm{CH}_{2} \mathrm{Cl}_{2}$ and hexanes were dried by passing through a column of activated alumina. Then, the solvents and $\mathrm{NEt}_{3}$ were dried by storing over activated $3 \AA$ molecular sieves in the glovebox. Commercially available $\mathrm{CD}_{2} \mathrm{Cl}_{2}$ (Cambridge Isotope Laboratories) was not distilled; instead it was simply dried over a large amount of activated $3 \AA$ molecular sieves in the glovebox. Bis(trifluoromethanesulfonyl)imide was purchased from Sigma-Aldrich.

\section{Reference:}

[33] R. K. Harris, E. D. Becker, S. M. Cabral de Menezes, R. Goodfellow, P. Granger, Pure Appl. Chem. 2001, 73, 1795-1818. 


\section{N-(Borabicyclo[3.3.1]nonan-9-yl)-1,1,1-trifluoro-N- (trifluoromethanesulfonyl)methanesulfonamide (5a)}

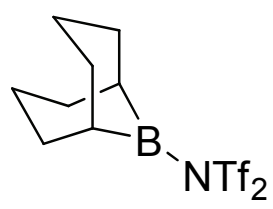

A suspension of 9-borabicyclo[3.3.1]nonane dimer (0.868 g, $7.11 \mathrm{mmol}$ of the monomer) and bis(trifluoromethanesulfonyl)imide $(2.00 \mathrm{~g}, 7.11 \mathrm{mmol})$ in $1 \mathrm{~mL}$ of dry toluene was carefully heated under a dry $\mathrm{N}_{2}$ atmosphere at $90{ }^{\circ} \mathrm{C}$ for 40 minutes. Intensive gas liberation was observed while heating, and a clear solution was formed. The resulting solution was distilled in vacuum, and a fraction boiling at $108{ }^{\circ} \mathrm{C}$ (1.5 Torr) was collected. Boron bistriflimide $\mathbf{5 a}$ is a very dense $\left(\mathrm{d}=1.49 \mathrm{~g} / \mathrm{cm}^{3}\right)$, viscous liquid and is highly air-sensitive.

${ }^{1} \mathrm{H}$ NMR (400 MHz, $\left.\mathrm{CD}_{2} \mathrm{Cl}_{2}, 25{ }^{\circ} \mathrm{C}, \mathrm{TMS}\right): \delta=2.07-1.81(\mathrm{~m}, 10 \mathrm{H}), 1.59-1.52(\mathrm{~m}, 2 \mathrm{H})$, 1.52-1.42 ppm (m, 2H). ${ }^{11} \mathrm{~B}$ NMR (128 MHz, $\left.\mathrm{CD}_{2} \mathrm{Cl}_{2}, \mathrm{Et}_{2} \mathrm{O}-\mathrm{BF}_{3}\right): \delta=59.2 \mathrm{ppm}(\mathrm{s}) .{ }^{13} \mathrm{C}$ NMR (101 MHz, $\left.\mathrm{CD}_{2} \mathrm{Cl}_{2}, \mathrm{TMS}\right): \delta=119.4$ (q, $\left.{ }^{1} J(\mathrm{C}-\mathrm{F})=325 \mathrm{~Hz}\right), 33.6,29.6-28.0(\mathrm{~m})$, 22.9 ppm. ${ }^{19} \mathrm{~F}$ NMR (377 MHz, $\mathrm{CD}_{2} \mathrm{Cl}_{2}, \mathrm{CFCl}_{3}$ ): $\delta=-70.0$ ppm (br s). HRMS (EI+ $70 \mathrm{eV}): \mathrm{m} / z$ : calculated 401.0362, found 401.0347 (-4 ppm). IR $\left(\mathrm{CCl}_{4}, \mathrm{CaF}_{2}\right): 1437,1417$, $1359,1325,1121 \mathrm{~cm}^{-1}$. 


\section{Preparation of Boronium Salt 8a}

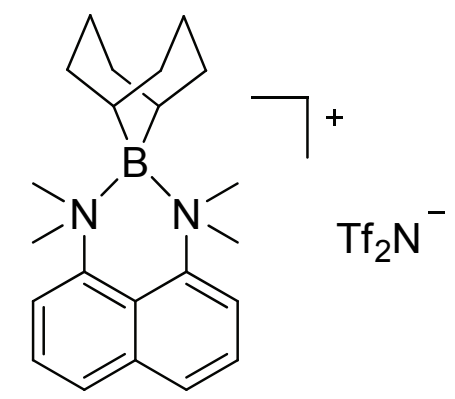

Method A. A mixture of 9-borabicyclo[3.3.1]nonane dimer $(0.461 \mathrm{~g}, 3.78 \mathrm{mmol}$ of the monomer) and bis(trifluoromethanesulfonyl)imide $(0.966 \mathrm{~g}, 3.44 \mathrm{mmol})$ in $5 \mathrm{~mL}$ of dry toluene was refluxed for 1 hour under nitrogen. Intensive gas liberation was observed while heating, and a clear solution was formed. The resulting solution along with $2 \times 1 \mathrm{~mL}$ of dry toluene was slowly added at room temperature to a solution of 1,8-bis(dimethylamino)naphthalene $(0.737 \mathrm{~g}, 3.44 \mathrm{mmol})$ in $3 \mathrm{~mL}$ of dry toluene. Upon mixing the reagents the reaction mixture developed a striking red color that persisted for a few seconds. A small exotherm was observed, and precipitation of a pale yellow oil began immediately. The oil crystallized within a few minutes of stirring at room temperature, the resulting crystals were isolated by filtration under nitrogen, and then washed with $2 \times 1 \mathrm{~mL}$ of dry toluene followed by $2 \mathrm{~mL}$ of dry hexanes. Drying the resulting solid in the glovebox yielded $2.04 \mathrm{~g}(96 \%)$ of the desired product. The resulting crystals of 8a are very stable in dry air, although solutions of the product are very sensitive.

Method B. Alternatively, the boronium salt 8a can be prepared by mixing equimolar amounts of the boron bis-triflimide reagent 5a and 1,8-bis(dimethylamino)-naphthalene in dry $\mathrm{CH}_{2} \mathrm{Cl}_{2}$ followed by evaporation of the solvent. The reaction was essentially instantaneous, but the product prepared in this manner was contaminated with the bis(trifluoromethanesulfonyl)imide salt of 1,8-bis(dimethylamino)naphthalene due to impurities in the boron reagent $5 \mathbf{a}$. 
8a: ${ }^{1} \mathrm{H}$ NMR (500 MHz, $\left.\mathrm{CD}_{2} \mathrm{Cl}_{2}, 25{ }^{\circ} \mathrm{C}, \mathrm{TMS}\right): \delta=8.01(\mathrm{dd}, J=8.3 \mathrm{~Hz}, 0.5 \mathrm{~Hz}, 2 \mathrm{H})$, 7.90 (dd, $J=8.0 \mathrm{~Hz}, 0.5 \mathrm{~Hz}, 2 \mathrm{H}), 7.74$ (t, $J=8.0 \mathrm{~Hz}, 2 \mathrm{H}), 3.48$ (s, 12H), 2.31-2.13 (m, $4 \mathrm{H}), 2.19-2.05(\mathrm{~m}, 2 \mathrm{H}), 1.81-1.67(\mathrm{~m}, 6 \mathrm{H}), 1.02-0.91 \mathrm{ppm}(\mathrm{br} \mathrm{s}, 2 \mathrm{H}) .{ }^{11} \mathrm{~B}$ NMR (128 $\left.\mathrm{MHz}, \mathrm{CD}_{2} \mathrm{Cl}_{2}, \mathrm{Et}_{2} \mathrm{O}-\mathrm{BF}_{3}\right): \delta=16.2 \mathrm{ppm}(\mathrm{s}) .{ }^{13} \mathrm{C} \mathrm{NMR}\left(101 \mathrm{MHz}, \mathrm{CD}_{2} \mathrm{Cl}_{2}, \mathrm{TMS}\right): \delta=$ $142.9,135.0,129.5,127.7,120.3\left(\mathrm{q},{ }^{1} J(\mathrm{C}-\mathrm{F})=322 \mathrm{~Hz}\right), 120.1,119.7,57.1,34.6,21.0-$ 19.9 (m), 20.3 ppm. ${ }^{19} \mathrm{~F}$ NMR (377 MHz, $\mathrm{CD}_{2} \mathrm{Cl}_{2}, \mathrm{CFCl}_{3}$ ): $\delta=-79.4$ ppm (s). HRMS (ES+): $m / z$ : calculated 335.2653, found 355.3655 (+1 ppm). $\operatorname{IR}\left(\mathrm{CD}_{2} \mathrm{Cl}_{2}, \mathrm{CaF}_{2}\right): 2874$, 1603, 1576, 1492, 1435, 1342, $1142 \mathrm{~cm}^{-1}$. m.p. $128{ }^{\circ} \mathrm{C}$ (sealed capillary); decomposition begins ca. $100{ }^{\circ} \mathrm{C}$. 


\section{Generation of Borenium Salt 10}

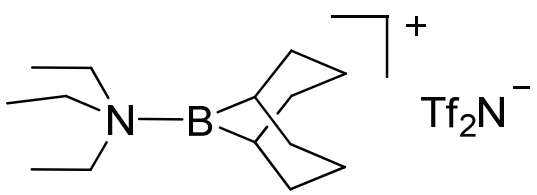

A dry J. Young NMR tube was charged with a solution of boron bis-triflimide 5a (30.0 $\mu \mathrm{L}, 44.7 \mathrm{mg}, 0.111 \mathrm{mmol})$ in $0.6 \mathrm{~mL}$ of dry $\mathrm{CD}_{2} \mathrm{Cl}_{2}$. Neat triethylamine $(15.5 \mu \mathrm{L}, 11.3$ $\mathrm{mg}, 0.112 \mathrm{mmol}$ ) was added, and the mixture was shaken vigorously. NMR analysis indicated clean formation of borenium salt $\mathbf{1 0}$.

10: ${ }^{1} \mathrm{H}$ NMR (400 MHz, $\mathrm{CD}_{2} \mathrm{Cl}_{2}, 25{ }^{\circ} \mathrm{C}$, TMS): $\delta=3.51$ (q, $J=7.4 \mathrm{~Hz}, 6 \mathrm{H}$ ), 2.35-2.26 (m, 4H), 2.25-2.12 (m, 2H), 2.07-1.94 (m, 4H), 1.87-1.80 (m, 2H), 1.66-1.56 (m, 2H), $1.35 \mathrm{ppm}(\mathrm{t}, J=7.4 \mathrm{~Hz}, 9 \mathrm{H}) .{ }^{11} \mathrm{~B}$ NMR (128 MHz, $\left.\mathrm{CD}_{2} \mathrm{Cl}_{2}, \mathrm{Et}_{2} \mathrm{O}-\mathrm{BF}_{3}\right): \delta=85.1 \mathrm{ppm}$ (s).

${ }^{13} \mathrm{C}$ NMR (101 MHz, $\left.\mathrm{CD}_{2} \mathrm{Cl}_{2}, \mathrm{TMS}\right): \delta=120.4\left(\mathrm{q},{ }^{1} J(\mathrm{C}-\mathrm{F})=322 \mathrm{~Hz}\right), 50.0,36.3$, 30.429.1 (m), 22.5, 9.6 ppm. $\left.{ }^{19} \mathrm{~F} \mathrm{NMR} \mathrm{(377} \mathrm{MHz,} \mathrm{CD}_{2} \mathrm{Cl}_{2}, \mathrm{CFCl}_{3}\right): \delta=-79.5 \mathrm{ppm}(\mathrm{s})$. 


\section{Preparation of Amine-Borane Complex 12}

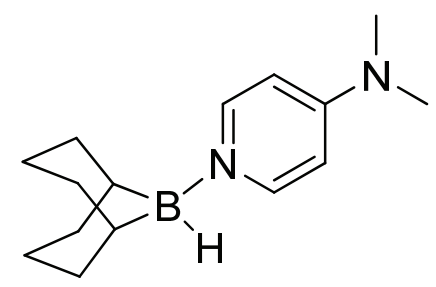

A mixture of solid 9-borabicyclo[3.3.1]nonane dimer $(82.2 \mathrm{mg}, 0.674 \mathrm{mmol}$ of the monomer) and 4-(dimethylamino)pyridine $(82.3 \mathrm{mg}, 0.674 \mathrm{mmol})$ was dissolved in $1 \mathrm{~mL}$ of dry $\mathrm{CH}_{2} \mathrm{Cl}_{2}$, and the resulting clear solution was stirred at room temperature overnight. A white precipitate appeared on stirring, and the slurry was concentrated to afford $\mathbf{1 2}$ as a white solid in quantitative yield.

12: ${ }^{1} \mathrm{H}$ NMR (500 MHz, $\left.\mathrm{CD}_{2} \mathrm{Cl}_{2}, 25^{\circ} \mathrm{C}, \mathrm{TMS}\right): \delta=8.11-8.06(\mathrm{~m}, 2 \mathrm{H}), 6.62-6.58(\mathrm{~m}, 2 \mathrm{H})$, 3.09 (s, 6H), 2.77-2.00 (m, 1H), 1.96-1.72 (m, 6H), 1.56-1.48 (m, 3H), 1.48-1.39 (m, $2 \mathrm{H}), 1.29-1.21(\mathrm{~m}, 1 \mathrm{H}), 1.13-1.04 \mathrm{ppm}(\mathrm{m}, 2 \mathrm{H}) .{ }^{11} \mathrm{~B} \mathrm{NMR}\left(128 \mathrm{MHz}, \mathrm{CD}_{2} \mathrm{Cl}_{2}, \mathrm{Et}_{2} \mathrm{O}-\right.$ $\left.\mathrm{BF}_{3}\right): \delta=-3.2 \mathrm{ppm}\left(\mathrm{d},{ }^{1} J(\mathrm{~B}-\mathrm{H})=60 \mathrm{~Hz}\right) \cdot{ }^{13} \mathrm{C} \mathrm{NMR}\left(101 \mathrm{MHz}, \mathrm{CD}_{2} \mathrm{Cl}_{2}, \mathrm{TMS}\right): \delta=155.1$, 144.8, 106.9, 39.6, 35.4, 29.5, 25.9, 25.5, 24.6-23.0 ppm (m). $\operatorname{IR}\left(\mathrm{CD}_{2} \mathrm{Cl}_{2}, \mathrm{CaF}_{2}\right): 2269$, $2239,1635,1547,1444,1344,1229,1202 \mathrm{~cm}^{-1}$. The compound has no distinct melting point; partial melting ca. $163{ }^{\circ} \mathrm{C}$ (sealed capillary) with decomposition. 


\section{Generation of Borenium Salt 13 and Boronium Salt 14}

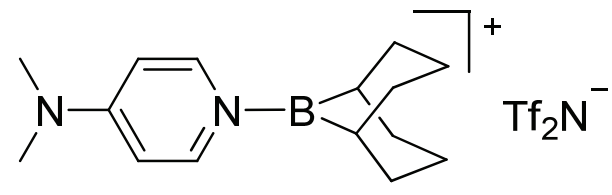

13

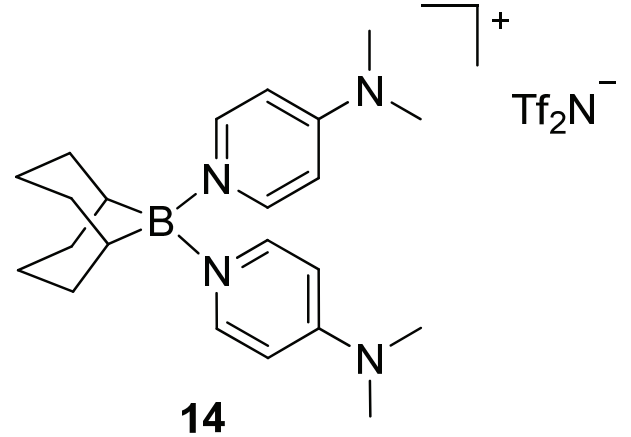

Solid bis(trifluoromethanesulfonyl)imide $(23.8 \mathrm{mg}, 84.8 \mu \mathrm{mol})$ was added in small portions to a stirred solution of amine-borane complex $12(20.7 \mathrm{mg}, 84.8 \mu \mathrm{mol})$ in $1 \mathrm{~mL}$ of dry $\mathrm{CD}_{2} \mathrm{Cl}_{2}$. Intensive gas liberation was observed. NMR assay of the resulting clear solution showed formation of borenium salt 13, along with minor amounts of the boronium salt 14 and DMAP·HNTf 2 (typically 7-11:1 13:14). Addition of an extra equivalent of 4-(dimethylamino)pyridine $(10.4 \mathrm{mg}, 84.8 \mu \mathrm{mol})$ cleanly produced boronium salt 14, as evidenced by NMR spectroscopy.

Alternatively, complex 14 was prepared by carefully treating a solution of 4(dimethylamino)pyridine (27.1 mg, $0.222 \mathrm{mmol}$ ) in $0.5 \mathrm{~mL}$ of anhydrous $\mathrm{CH}_{2} \mathrm{Cl}_{2}$ with neat boron bis-triflimide 5a $(30.0 \mu \mathrm{L}, 44.7 \mathrm{mg}, 0.111 \mathrm{mmol})$. Concentration of the resulting solution afforded a white crystalline solid in quantitative yield.

13: ${ }^{1} \mathrm{H}$ NMR (500 MHz, $\left.\mathrm{CD}_{2} \mathrm{Cl}_{2}, 2{ }^{\circ} \mathrm{C}, \mathrm{TMS}\right)$ : $\delta=8.37-8.33$ (m, 2H), 6.99-6.95 (m, 2H), $3.37(\mathrm{~s}, 6 \mathrm{H}), 2.14-1.98(\mathrm{~m}, 8 \mathrm{H}), 1.93-1.84(\mathrm{~m}, 4 \mathrm{H}), 1.45-1.37 \mathrm{ppm}(\mathrm{m}, 2 \mathrm{H}) .{ }^{11} \mathrm{~B}$ NMR (128 MHz, $\left.\mathrm{CD}_{2} \mathrm{Cl}_{2}, \mathrm{Et}_{2} \mathrm{O}-\mathrm{BF}_{3}\right): \delta=66.5 \mathrm{ppm}$ (s). ${ }^{13} \mathrm{C} \mathrm{NMR}$ (101 MHz, $\left.\mathrm{CD}_{2} \mathrm{Cl}_{2}, \mathrm{TMS}\right): \delta$ $=159.2,142.6,120.3\left(\mathrm{q},{ }^{1} J(\mathrm{C}-\mathrm{F})=322 \mathrm{~Hz}\right), 108.8,41.2,34.4,28.0-26.2(\mathrm{~m}), 23.2 \mathrm{ppm}$. ${ }^{19} \mathrm{~F} \mathrm{NMR}\left(377 \mathrm{MHz}, \mathrm{CD}_{2} \mathrm{Cl}_{2}, \mathrm{CFCl}_{3}\right): \delta=-79.4 \mathrm{ppm}(\mathrm{s})$.

14: ${ }^{1} \mathrm{H}$ NMR (500 MHz, $\mathrm{CD}_{2} \mathrm{Cl}_{2}, 2{ }^{\circ} \mathrm{C}$, TMS): $\delta=8.07-8.03(\mathrm{~m}, 4 \mathrm{H}), 6.69-6.65(\mathrm{~m}, 4 \mathrm{H})$, $3.11(\mathrm{~s}, 12 \mathrm{H}), 1.97-1.85(\mathrm{~m}, 2 \mathrm{H}), 1.85-1.78(\mathrm{~m}, 4 \mathrm{H}), 1.62-1.58(\mathrm{~m}, 2 \mathrm{H}), 1.58-1.48(\mathrm{~m}$, $4 \mathrm{H}), 1.38-1.31 \mathrm{ppm}(\mathrm{m}, 2 \mathrm{H}) .{ }^{11} \mathrm{~B} \mathrm{NMR}\left(128 \mathrm{MHz}, \mathrm{CD}_{2} \mathrm{Cl}_{2}, \mathrm{Et}_{2} \mathrm{O}-\mathrm{BF}_{3}\right): \delta=3.0 \mathrm{ppm}(\mathrm{s})$. ${ }^{13} \mathrm{C}$ NMR (101 MHz, $\left.\mathrm{CD}_{2} \mathrm{Cl}_{2}, \mathrm{TMS}\right): \delta=156.3,142.8,120.4\left(\mathrm{q},{ }^{1} J(\mathrm{C}-\mathrm{F})=322 \mathrm{~Hz}\right)$, 108.1, 39.9, 30.4, 23.7, 21.5-20.5 ppm (m). ${ }^{19} \mathrm{~F}$ NMR (377 MHz, $\mathrm{CD}_{2} \mathrm{Cl}_{2}, \mathrm{CFCl}_{3}$ ): 
$\delta=-79.5 \mathrm{ppm}(\mathrm{s})$. HRMS (ES+): $\mathrm{m} / \mathrm{z}$ : calculated 365.2871, found $365.2873(+1 \mathrm{ppm})$. $\operatorname{IR}\left(\mathrm{CD}_{2} \mathrm{Cl}_{2}, \mathrm{CaF}_{2}\right): 2891,2851,1635,1557,1444,1350,1138 \mathrm{~cm}^{-1}$. The compound has no distinct melting point; partial melting ca. $208^{\circ} \mathrm{C}$ (sealed capillary) with decomposition. UV/Vis $\left(\mathrm{CH}_{2} \mathrm{Cl}_{2}\right)$ : $\lambda_{\max }(\varepsilon)=295$ (24000), $283 \mathrm{~nm}(21000)$. 


\section{Representative Procedure for Indole and Pyrrole Borylation}

A dry $4 \mathrm{~mL}$ scintillation vial was charged with a solution of the desired substrate $(0.16$ $0.17 \mathrm{mmol}$ ) and boronium salt 8a (1.05 equivalents per each 9-BBN unit introduced) in dry $\mathrm{CH}_{2} \mathrm{Cl}_{2}$ (0.5 mL per each 9-BBN unit introduced). The reaction vessel was sealed, heated at $50{ }^{\circ} \mathrm{C}$ for the indicated amount of time, and the reaction mixture was then diluted with dry hexanes (1 mL per each 9-BBN unit introduced) and left at room temperature for 1-2 hours to allow the byproduct to precipitate. Following decantation of supernatant, the solids were washed with $4 \times 0.5 \mathrm{~mL}$ of dry hexanes, and the combined extracts were evaporated to dryness to give crude material. The pure product was obtained by extracting the crude material with $4 \times 0.5 \mathrm{~mL}$ of dry hexanes followed by concentration of the extracts. All of the resulting borylated heterocycles were found to be extremely sensitive to water and were protected from exposure to air by working under dry nitrogen. UV spectra of the borylated heterocycles were acquired in $0.83 \%(\mathrm{v} / \mathrm{v})$ solution of $\mathrm{NEt}_{3}$ in hexanes. Very rapid protodeboronation precludes acquisition of UV spectra in the absence of $\mathrm{NEt}_{3}$.

\section{3-(9-Borabicyclo[3.3.1]nonan-9-yl)-1-methyl-1H-indole (16a)}

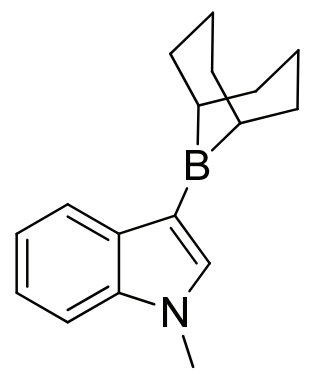

Reaction time 1.5 hours, $96 \%$ yield of a colorless crystalline solid.

${ }^{1} \mathrm{H}$ NMR (500 MHz, $\left.\mathrm{CD}_{2} \mathrm{Cl}_{2}, 25{ }^{\circ} \mathrm{C}, \mathrm{TMS}\right): \delta=8.04(\mathrm{~d}, J=8.0 \mathrm{~Hz}, 1 \mathrm{H}), 7.74(\mathrm{~s}, 1 \mathrm{H})$, $7.37(\mathrm{~d}, J=8.0 \mathrm{~Hz}, 1 \mathrm{H}), 7.27-7.23(\mathrm{~m}, 1 \mathrm{H}), 7.20-7.16(\mathrm{~m}, 1 \mathrm{H}), 3.83$ (s, 3H), 2.38-2.29 $(\mathrm{m}, 2 \mathrm{H}), 2.07-1.95(\mathrm{~m}, 6 \mathrm{H}), 1.94-1.82(\mathrm{~m}, 4 \mathrm{H}), 1.40-1.31 \mathrm{ppm}(\mathrm{m}, 2 \mathrm{H}) .{ }^{11} \mathrm{~B}$ NMR (128 $\left.\mathrm{MHz}, \mathrm{CD}_{2} \mathrm{Cl}_{2}, \mathrm{Et}_{2} \mathrm{O}-\mathrm{BF}_{3}\right): \delta=72.6 \mathrm{ppm}(\mathrm{s}) .{ }^{13} \mathrm{C} \mathrm{NMR}\left(101 \mathrm{MHz}, \mathrm{CD}_{2} \mathrm{Cl}_{2}, \mathrm{TMS}\right): \delta=$ 
$141.7,139.8,133.5,122.9,122.2,121.2,116.2-114.9$ (m), 110.0, 34.3, 33.5, 30.0-28.8 (m), $24.0 \mathrm{ppm}$. HRMS (EI+ $70 \mathrm{eV}): \mathrm{m} / z$ : calculated 251.1845, found $251.1850(+2 \mathrm{ppm})$. $\operatorname{IR}\left(\mathrm{CCl}_{4}, \mathrm{CaF}_{2}\right): 1675,1511,1465,1421,1364,1333,1159,1132,1109 \mathrm{~cm}^{-1}$. m.p. $106-108{ }^{\circ} \mathrm{C}$ (sealed capillary). UV/Vis $\left(0.83 \% \mathrm{v} / \mathrm{v} \mathrm{NEt}_{3}\right.$ in hexanes): $\lambda_{\max }(\varepsilon)=291 \mathrm{~nm}$ (15000).

Compound 16a was also prepared on a larger scale using a modified procedure. A dry 12 $\mathrm{mL}$ thick-walled Schlenk tube fitted with a teflon stopper was charged with a mixture of $\mathrm{N}$-methylindole (206 $\mu \mathrm{L}, 0.218 \mathrm{~g}, 1.66 \mathrm{mmol})$ and boronium salt 8a (1.07 g, $1.74 \mathrm{mmol})$ in $3 \mathrm{~mL}$ of dry $\mathrm{CH}_{2} \mathrm{Cl}_{2}$. The reaction vessel was sealed and then heated at $50{ }^{\circ} \mathrm{C}$ for $2 \mathrm{~h}$. The reaction mixture was then diluted with $5 \mathrm{~mL}$ of dry hexanes and left at room temperature for 1-2 hours to allow the byproduct to precipitate. Following decantation of supernatant, the solids were washed with $3 \times 1 \mathrm{~mL}$ of dry hexanes, and the combined extracts were evaporated to dryness to give crude material. The pure product $(0.415 \mathrm{~g})$ was obtained in essentially quantitative yield by extracting the crude material with 5 portions of dry hexanes (total solvent volume $12 \mathrm{~mL}$ ) followed by concentration of the extracts.

\section{3-(9-Borabicyclo[3.3.1]nonan-9-yl)-1,2,5-trimethyl-1H-pyrrole (16b)}

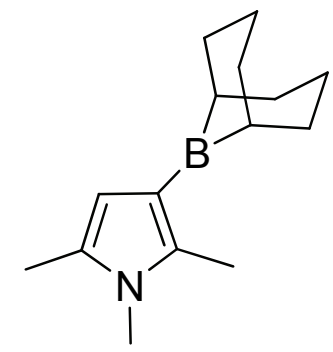

Reaction time 1.5 hours, $98 \%$ yield of a colorless crystalline solid.

${ }^{1} \mathrm{H}$ NMR (500 MHz, $\left.\mathrm{CD}_{2} \mathrm{Cl}_{2}, 25{ }^{\circ} \mathrm{C}, \mathrm{TMS}\right): \delta=6.21$ (q, $\left.J=0.9 \mathrm{~Hz}, 1 \mathrm{H}\right), 3.41(\mathrm{~s}, 3 \mathrm{H})$, $2.43(\mathrm{~s}, 3 \mathrm{H}), 2.20$ (s, 3H), 2.11-2.05 (m, 2H), 2.01-1.86 (m, 6H), 1.82-1.73 (m, 4H), 1.35$1.25 \mathrm{ppm}(\mathrm{m}, 2 \mathrm{H}) .{ }^{11} \mathrm{~B} \mathrm{NMR}\left(128 \mathrm{MHz}, \mathrm{CD}_{2} \mathrm{Cl}_{2}, \mathrm{Et}_{2} \mathrm{O}-\mathrm{BF}_{3}\right): \delta=72.2 \mathrm{ppm}(\mathrm{s}) .{ }^{13} \mathrm{C} \mathrm{NMR}$ (101 MHz, $\left.\mathrm{CD}_{2} \mathrm{Cl}_{2}, \mathrm{TMS}\right): \delta=140.5,129.0,120.0-118.3$ (m), 112.7, 34.2, 30.6, 29.8- 
$29.0(\mathrm{~m}), 24.0,13.4,12.6 \mathrm{ppm}$. HRMS (EI+ $70 \mathrm{eV}): \mathrm{m} / \mathrm{z}$ : calculated 229.2002, found 229.2002 (0 ppm). IR( $\left(\mathrm{CCl}_{4}, \mathrm{CaF}_{2}\right): 2915,2837,1503,1433,1405,1372,1350,1172$, $1115 \mathrm{~cm}^{-1}$. m.p. $120^{\circ} \mathrm{C}$ (sealed capillary). UV/Vis $\left(0.83 \% \mathrm{v} / \mathrm{v} \mathrm{NEt} t_{3}\right.$ in hexanes): $\lambda_{\max }(\varepsilon)=$ $286 \mathrm{~nm}(6100)$.

\section{2,4-Di(9-borabicyclo[3.3.1] nonan-9-yl)-1-methyl-1 $H$-pyrrole (16c)}

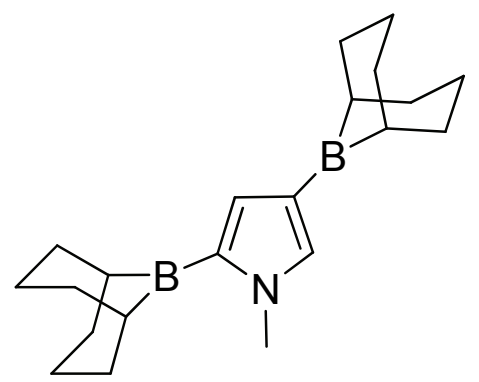

Reaction time 3.5 hours, $97 \%$ yield of a colorless crystalline solid.

${ }^{1} \mathrm{H}$ NMR (500 MHz, $\left.\mathrm{CD}_{2} \mathrm{Cl}_{2}, 25^{\circ} \mathrm{C}, \mathrm{TMS}\right): \delta=7.60(\mathrm{~d}, J=1.6 \mathrm{~Hz}, 1 \mathrm{H}), 7.55$ (dd, $J=1.6$ $\mathrm{Hz}, 0.4 \mathrm{~Hz}, 1 \mathrm{H}), 3.92$ (t, $J=0.4 \mathrm{~Hz}, 3 \mathrm{H}), 2.23-2.18$ (m, 2H), 2.08-1.90 (m, 14H), 1.90$1.73(\mathrm{~m}, 8 \mathrm{H}), 1.41-1.25 \mathrm{ppm}(\mathrm{m}, 4 \mathrm{H}) .{ }^{11} \mathrm{~B}$ NMR (128 MHz, $\left.\mathrm{CD}_{2} \mathrm{Cl}_{2}, \mathrm{Et}_{2} \mathrm{O}-\mathrm{BF}_{3}\right): \delta=73.0$ (s), $70.2 \mathrm{ppm}(\mathrm{s}) .{ }^{13} \mathrm{C} \mathrm{NMR}$ (101 MHz, $\left.\mathrm{CD}_{2} \mathrm{Cl}_{2}, \mathrm{TMS}\right): \delta=142.8,141.2-140.0(\mathrm{~m})$, 134.4, 124.0-123.1 (m), 38.4, 34.4, 34.3, 29.9-29.1(m), 29.1-28.3 (m), 24.0, 23.8 ppm. HRMS (EI+ $70 \mathrm{eV}): m / z$ : calculated 321.2799, found 321.2811 (+4 ppm). $\operatorname{IR}\left(\mathrm{CCl}_{4}\right.$, $\mathrm{CaF}_{2}$ ): 1656, 1486, 1450,1422, 1362, 1325, 1150, $1112 \mathrm{~cm}^{-1}$. m.p. $133-135^{\circ} \mathrm{C}$ (sealed capillary). UV/Vis $\left(0.83 \% \mathrm{v} / \mathrm{v} \mathrm{NEt}{ }_{3}\right.$ in hexanes): $\lambda_{\max }(\varepsilon)=290 \mathrm{~nm}(22000)$. 


\section{1,3-Di(9-borabicyclo[3.3.1]nonan-9-yl)-1H-indole (16d)}

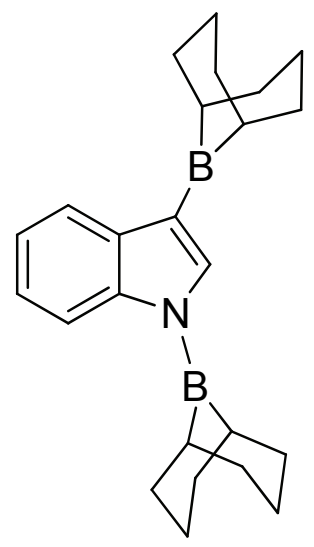

Reaction time 5.5 days, $97 \%$ yield of a colorless crystalline solid.

${ }^{1} \mathrm{H}$ NMR (500 MHz, $\left.\mathrm{CD}_{2} \mathrm{Cl}_{2}, 25{ }^{\circ} \mathrm{C}, \mathrm{TMS}\right): \delta=8.22$ (s, $\left.1 \mathrm{H}\right), 8.03-7.99(\mathrm{~m}, 1 \mathrm{H}), 7.93-$ $7.88(\mathrm{~m}, 1 \mathrm{H}), 7.28-7.23(\mathrm{~m}, 2 \mathrm{H}), 2.70-2.21(\mathrm{~m}, 4 \mathrm{H}), 2.16-1.83(\mathrm{~m}, 20 \mathrm{H}), 1.50-1.41(\mathrm{~m}$, 2H), 1.41-1.32 ppm (m, 2H). ${ }^{11} \mathrm{~B}$ NMR (128 MHz, $\left.\mathrm{CD}_{2} \mathrm{Cl}_{2}, \mathrm{Et}_{2} \mathrm{O}-\mathrm{BF}_{3}\right): \delta=75.7$ (s), 62.0 ppm (s). ${ }^{13} \mathrm{C}$ NMR (101 MHz, $\left.\mathrm{CD}_{2} \mathrm{Cl}_{2}, \mathrm{TMS}\right): \delta=143.5,143.3,137.4,123.5,123.2$, 122.9, 122.7-122.0 (m), 116.0, 34.3, 34.1, 30.7-29.6 (m), 28.2-26.3 (m), 23.9, 23.5 ppm. HRMS $(\mathrm{EI}+70 \mathrm{eV}): \mathrm{m} / \mathrm{z}$ : calculated 357.2799, found 357.2814 (+4 ppm). IR( $\mathrm{CCl}_{4}$, $\left.\mathrm{CaF}_{2}\right): 1694,1487,1471,1449,1405,1334,1292,1135,1108 \mathrm{~cm}^{-1}$. m.p. 204-206 ${ }^{\circ} \mathrm{C}$ (sealed capillary). 


\section{X-ray Crystallography Details of 8a}

Colorless plates of 8a were grown from a hexanes/dichloromethane solution by slow cooling to ambient temperature. A crystal of dimensions $0.37 \times 0.37 \times 0.025 \mathrm{~mm}$ was mounted on a Bruker SMART APEX CCD-based X-ray diffractometer equipped with a low temperature device and fine focus Mo-target X-ray tube $(\lambda=0.71073 \AA)$ operated at $1500 \mathrm{~W}$ power $(50 \mathrm{kV}, 30 \mathrm{~mA})$. The X-ray intensities were measured at $85(1) \mathrm{K}$; the detector was placed at a distance $5.055 \mathrm{~cm}$ from the crystal. A total of 2790 frames were collected with a scan width of $0.5^{\circ}$ in $\omega$ and $0.45^{\circ}$ in $\varphi$ with an exposure time of $25 \mathrm{~s} /$ frame. The integration of the data yielded a total of 61663 reflections to a maximum $2 \theta$ value of $56.58^{\circ}$ of which 6437 were independent and 5096 were greater than $2 \sigma(I)$. The final cell constants (monoclinic, $\mathrm{a}=12.0734(12) \AA ; \alpha=90^{\circ} ; \mathrm{b}=26.951(3) \AA ; \beta=$ $100.946(2)^{\circ} ; \mathrm{c}=8.1362(8) \AA ; \gamma=90^{\circ} ; \mathrm{V}=2599.2(4) \AA^{3}$ ) were based on the $\mathrm{xyz}$ centroids of 9873 reflections above $10 \sigma(\mathrm{I})$. Linear absorption coefficient $\mu=0.287 \mathrm{~mm}^{-1}$. Analysis of the data showed negligible decay during data collection; the data were processed with SADABS and corrected for absorption. The structure was solved and refined with the Bruker SHELXTL (version 2008/3) software package, using the space group P2(1)/c with $\mathrm{Z}=4$ for the formula $\mathrm{C}_{24} \mathrm{H}_{32} \mathrm{BF}_{6} \mathrm{~N}_{3} \mathrm{O}_{4} \mathrm{~S}_{2}\left(\mathrm{M}_{\mathrm{r}} 615.46 \mathrm{~g} / \mathrm{mol}\right.$, $\rho_{\text {calcd }}=$ $\left.1.573 \mathrm{~g} / \mathrm{cm}^{3}\right)$. All non-hydrogen atoms were refined anisotropically with the hydrogen atoms placed in idealized positions. Full matrix least-squares refinement based on $\mathrm{F}^{2}$ converged at $\mathrm{R} 1=0.0493$ and $\mathrm{wR} 2=0.1158$ [based on $\mathrm{I}>2 \sigma(\mathrm{I})], \mathrm{R} 1=0.0671$ and $\mathrm{wR} 2$ $=0.1255$ for all data. CCDC-791459 contains the supplementary crystallographic data for 8a. The cif file can be obtained free of charge from Cambridge Crystallographic Data Center (http://www.ccdc.cam.ac.uk/products/csd/request/).

Sheldrick, G.M. SHELXTL, v. 2008/3, Bruker Analytical X-ray, Madison, WI, 2008; Sheldrick, G.M. SADABS, v. 2008/1, Program for Empirical Absorption Correction of Area Detector Data, University of Gottingen, Gottingen, Germany, 2008; Saint Plus, v. 7.53a, Bruker Analytical X-ray, Madison, WI, 2008 


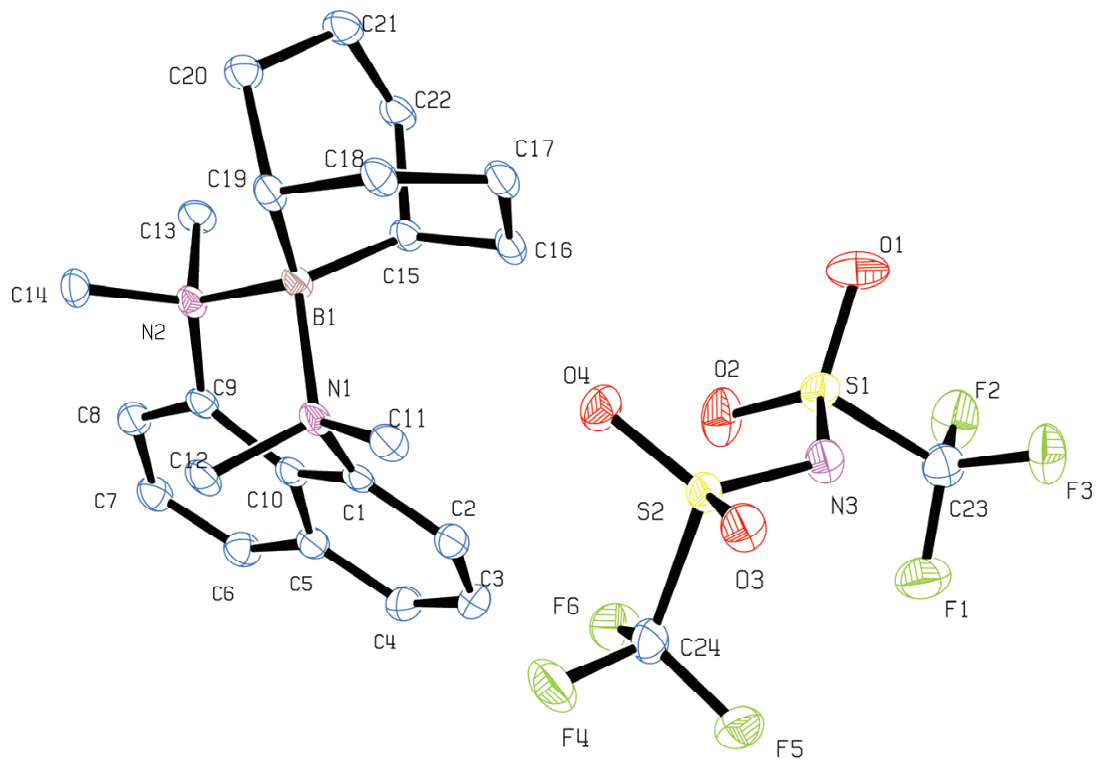

Bond lengths, Å:

\begin{tabular}{c|c|}
\hline $\mathrm{B}(1)-\mathrm{C}(19)$ & $1.612(3)$ \\
\hline $\mathrm{B}(1)-\mathrm{C}(15)$ & $1.631(3)$ \\
\hline $\mathrm{B}(1)-\mathrm{N}(1)$ & $1.724(3)$ \\
\hline $\mathrm{B}(1)-\mathrm{N}(2)$ & $1.732(3)$ \\
\hline $\mathrm{N}(1)-\mathrm{C}(1)$ & $1.477(3)$ \\
\hline $\mathrm{N}(1)-\mathrm{C}(11)$ & $1.513(2)$ \\
\hline $\mathrm{N}(1)-\mathrm{C}(12)$ & $1.523(2)$ \\
\hline $\mathrm{N}(2)-\mathrm{C}(9)$ & $1.511(3)$ \\
\hline $\mathrm{N}(2)-\mathrm{C}(14)$ & $1.522(2)$ \\
\hline $\mathrm{N}(2)-\mathrm{C}(13)$ & $1.525(2)$ \\
\hline $\mathrm{N}(3)-\mathrm{S}(2)$ & $1.5690(18)$ \\
\hline $\mathrm{N}(3)-\mathrm{S}(1)$ & $1.5923(18)$ \\
\hline $\mathrm{C}(1)-\mathrm{C}(2)$ & $1.370(3)$ \\
\hline $\mathrm{C}(1)-\mathrm{C}(10)$ & $1.423(3)$ \\
\hline $\mathrm{C}(2)-\mathrm{C}(3)$ & $1.413(3)$ \\
\hline
\end{tabular}

\begin{tabular}{|l|l|}
\hline $\mathrm{C}(3)-\mathrm{C}(4)$ & $1.362(3)$ \\
\hline $\mathrm{C}(4)-\mathrm{C}(5)$ & $1.421(3)$ \\
\hline $\mathrm{C}(5)-\mathrm{C}(6)$ & $1.419(3)$ \\
\hline $\mathrm{C}(5)-\mathrm{C}(10)$ & $1.429(3)$ \\
\hline $\mathrm{C}(6)-\mathrm{C}(7)$ & $1.368(3)$ \\
\hline $\mathrm{C}(7)-\mathrm{C}(8)$ & $1.408(3)$ \\
\hline $\mathrm{C}(8)-\mathrm{C}(9)$ & $1.376(3)$ \\
\hline $\mathrm{C}(9)-\mathrm{C}(10)$ & $1.426(3)$ \\
\hline $\mathrm{C}(15)-\mathrm{C}(16)$ & $1.542(3)$ \\
\hline $\mathrm{C}(15)-\mathrm{C}(22)$ & $1.565(3)$ \\
\hline $\mathrm{C}(16)-\mathrm{C}(17)$ & $1.543(3)$ \\
\hline $\mathrm{C}(17)-\mathrm{C}(18)$ & $1.556(3)$ \\
\hline $\mathrm{C}(18)-\mathrm{C}(19)$ & $1.570(3)$ \\
\hline $\mathrm{C}(19)-\mathrm{C}(20)$ & $1.555(3)$ \\
\hline $\mathrm{C}(20)-\mathrm{C}(21)$ & $1.537(3)$ \\
\hline
\end{tabular}

\begin{tabular}{|l|l}
\hline $\mathrm{C}(21)-\mathrm{C}(22)$ & $1.543(3)$ \\
\hline $\mathrm{C}(23)-\mathrm{F}(1)$ & $1.328(3)$ \\
\hline $\mathrm{C}(23)-\mathrm{F}(3)$ & $1.331(2)$ \\
\hline $\mathrm{C}(23)-\mathrm{F}(2)$ & $1.337(2)$ \\
\hline $\mathrm{C}(23)-\mathrm{S}(1)$ & $1.831(2)$ \\
\hline $\mathrm{C}(24)-\mathrm{F}(6)$ & $1.328(2)$ \\
\hline $\mathrm{C}(24)-\mathrm{F}(5)$ & $1.333(2)$ \\
\hline $\mathrm{C}(24)-\mathrm{F}(4)$ & $1.336(2)$ \\
\hline $\mathrm{C}(24)-\mathrm{S}(2)$ & $1.844(2)$ \\
\hline $\mathrm{S}(1)-\mathrm{O}(1)$ & $1.4311(17)$ \\
\hline $\mathrm{S}(1)-\mathrm{O}(2)$ & $1.4319(16)$ \\
\hline $\mathrm{S}(2)-\mathrm{O}(4)$ & $1.4357(15)$ \\
\hline $\mathrm{S}(2)-\mathrm{O}(3)$ & $1.4384(15)$ \\
\hline
\end{tabular}


Bond angles, deg.:

\begin{tabular}{l|l|}
\hline $\mathrm{C}(19)-\mathrm{B}(1)-\mathrm{C}(15)$ & $106.00(15)$ \\
\hline $\mathrm{C}(19)-\mathrm{B}(1)-\mathrm{N}(1)$ & $112.06(16)$ \\
\hline $\mathrm{C}(15)-\mathrm{B}(1)-\mathrm{N}(1)$ & $112.40(16)$ \\
\hline $\mathrm{C}(19)-\mathrm{B}(1)-\mathrm{N}(2)$ & $115.36(16)$ \\
\hline $\mathrm{C}(15)-\mathrm{B}(1)-\mathrm{N}(2)$ & $111.28(15)$ \\
\hline $\mathrm{N}(1)-\mathrm{B}(1)-\mathrm{N}(2)$ & $99.91(13)$ \\
\hline $\mathrm{C}(1)-\mathrm{N}(1)-\mathrm{C}(11)$ & $111.39(15)$ \\
\hline $\mathrm{C}(1)-\mathrm{N}(1)-\mathrm{C}(12)$ & $106.81(15)$ \\
\hline $\mathrm{C}(11)-\mathrm{N}(1)-\mathrm{C}(12)$ & $100.32(14)$ \\
\hline $\mathrm{C}(1)-\mathrm{N}(1)-\mathrm{B}(1)$ & $106.55(14)$ \\
\hline $\mathrm{C}(11)-\mathrm{N}(1)-\mathrm{B}(1)$ & $118.00(15)$ \\
\hline $\mathrm{C}(12)-\mathrm{N}(1)-\mathrm{B}(1)$ & $113.31(14)$ \\
\hline $\mathrm{C}(9)-\mathrm{N}(2)-\mathrm{C}(14)$ & $111.87(15)$ \\
\hline $\mathrm{C}(9)-\mathrm{N}(2)-\mathrm{C}(13)$ & $102.43(15)$ \\
\hline $\mathrm{C}(14)-\mathrm{N}(2)-\mathrm{C}(13)$ & $103.89(15)$ \\
\hline $\mathrm{C}(9)-\mathrm{N}(2)-\mathrm{B}(1)$ & $114.19(14)$ \\
\hline $\mathrm{C}(14)-\mathrm{N}(2)-\mathrm{B}(1)$ & $112.98(15)$ \\
\hline $\mathrm{C}(13)-\mathrm{N}(2)-\mathrm{B}(1)$ & $110.42(14)$ \\
\hline $\mathrm{S}(2)-\mathrm{N}(3)-\mathrm{S}(1)$ & $123.98(11)$ \\
\hline $\mathrm{C}(2)-\mathrm{C}(1)-\mathrm{C}(10)$ & $121.05(19)$ \\
\hline $\mathrm{C}(2)-\mathrm{C}(1)-\mathrm{N}(1)$ & $123.71(18)$ \\
\hline $\mathrm{C}(10)-\mathrm{C}(1)-\mathrm{N}(1)$ & $115.07(17)$ \\
\hline $\mathrm{C}(1)-\mathrm{C}(2)-\mathrm{C}(3)$ & $119.97(19)$ \\
\hline $\mathrm{C}(4)-\mathrm{C}(3)-\mathrm{C}(2)$ & $120.4(2)$ \\
\hline $\mathrm{C}(3)-\mathrm{C}(4)-\mathrm{C}(5)$ & $120.9(2)$ \\
\hline $\mathrm{C}(6)-\mathrm{C}(5)-\mathrm{C}(4)$ & $121.9(2)$ \\
\hline $\mathrm{C}(6)-\mathrm{C}(5)-\mathrm{C}(10)$ & $119.30(19)$ \\
\hline $\mathrm{C}(4)-\mathrm{C}(5)-\mathrm{C}(10)$ & $118.82(19)$ \\
\hline $\mathrm{C}(7)-\mathrm{C}(6)-\mathrm{C}(5)$ & $120.4(2)$ \\
\hline
\end{tabular}

\begin{tabular}{|l|l}
\hline $\mathrm{C}(16)-\mathrm{C}(15)-\mathrm{C}(22)$ & $106.72(16)$ \\
\hline $\mathrm{C}(16)-\mathrm{C}(15)-\mathrm{B}(1)$ & $109.06(16)$ \\
\hline $\mathrm{C}(22)-\mathrm{C}(15)-\mathrm{B}(1)$ & $114.17(16)$ \\
\hline $\mathrm{C}(15)-\mathrm{C}(16)-\mathrm{C}(17)$ & $113.07(17)$ \\
\hline $\mathrm{C}(16)-\mathrm{C}(17)-\mathrm{C}(18)$ & $117.65(16)$ \\
\hline $\mathrm{C}(17)-\mathrm{C}(18)-\mathrm{C}(19)$ & $118.33(16)$ \\
\hline $\mathrm{C}(20)-\mathrm{C}(19)-\mathrm{C}(18)$ & $106.63(15)$ \\
\hline $\mathrm{C}(20)-\mathrm{C}(19)-\mathrm{B}(1)$ & $110.84(16)$ \\
\hline $\mathrm{C}(18)-\mathrm{C}(19)-\mathrm{B}(1)$ & $111.40(16)$ \\
\hline $\mathrm{C}(21)-\mathrm{C}(20)-\mathrm{C}(19)$ & $112.51(17)$ \\
\hline $\mathrm{C}(20)-\mathrm{C}(21)-\mathrm{C}(22)$ & $116.00(17)$ \\
\hline $\mathrm{C}(21)-\mathrm{C}(22)-\mathrm{C}(15)$ & $117.23(17)$ \\
\hline $\mathrm{F}(1)-\mathrm{C}(23)-\mathrm{F}(3)$ & $108.08(18)$ \\
\hline $\mathrm{F}(1)-\mathrm{C}(23)-\mathrm{F}(2)$ & $108.12(19)$ \\
\hline $\mathrm{F}(3)-\mathrm{C}(23)-\mathrm{F}(2)$ & $108.31(18)$ \\
\hline $\mathrm{F}(1)-\mathrm{C}(23)-\mathrm{S}(1)$ & $111.61(15)$ \\
\hline $\mathrm{F}(3)-\mathrm{C}(23)-\mathrm{S}(1)$ & $111.86(16)$ \\
\hline $\mathrm{F}(2)-\mathrm{C}(23)-\mathrm{S}(1)$ & $108.74(15)$ \\
\hline $\mathrm{F}(6)-\mathrm{C}(24)-\mathrm{F}(5)$ & $108.09(18)$ \\
\hline $\mathrm{F}(6)-\mathrm{C}(24)-\mathrm{F}(4)$ & $108.31(17)$ \\
\hline $\mathrm{F}(5)-\mathrm{C}(24)-\mathrm{F}(4)$ & $107.72(17)$ \\
\hline $\mathrm{F}(6)-\mathrm{C}(24)-\mathrm{S}(2)$ & $112.58(15)$ \\
\hline $\mathrm{F}(5)-\mathrm{C}(24)-\mathrm{S}(2)$ & $110.91(14)$ \\
\hline $\mathrm{F}(4)-\mathrm{C}(24)-\mathrm{S}(2)$ & $109.10(15)$ \\
\hline $\mathrm{O}(1)-\mathrm{S}(1)-\mathrm{O}(2)$ & $118.22(11)$ \\
\hline $\mathrm{O}(1)-\mathrm{S}(1)-\mathrm{N}(3)$ & $112.11(10)$ \\
\hline $\mathrm{O}(2)-\mathrm{S}(1)-\mathrm{N}(3)$ & $115.72(10)$ \\
\hline $\mathrm{O}(1)-\mathrm{S}(1)-\mathrm{C}(23)$ & $104.34(11)$ \\
\hline $\mathrm{O}(2)-\mathrm{S}(1)-\mathrm{C}(23)$ & $105.43(10)$ \\
\hline
\end{tabular}




\begin{tabular}{l|l|}
\hline $\mathrm{C}(6)-\mathrm{C}(7)-\mathrm{C}(8)$ & $120.33(19)$ \\
\hline $\mathrm{C}(9)-\mathrm{C}(8)-\mathrm{C}(7)$ & $120.78(19)$ \\
\hline $\mathrm{C}(8)-\mathrm{C}(9)-\mathrm{C}(10)$ & $120.02(18)$ \\
\hline $\mathrm{C}(8)-\mathrm{C}(9)-\mathrm{N}(2)$ & $119.42(17)$ \\
\hline $\mathrm{C}(10)-\mathrm{C}(9)-\mathrm{N}(2)$ & $120.08(16)$ \\
\hline $\mathrm{C}(1)-\mathrm{C}(10)-\mathrm{C}(9)$ & $123.64(19)$ \\
\hline $\mathrm{C}(1)-\mathrm{C}(10)-\mathrm{C}(5)$ & $117.95(18)$ \\
\hline $\mathrm{C}(9)-\mathrm{C}(10)-\mathrm{C}(5)$ & $118.29(18)$ \\
\hline
\end{tabular}

\begin{tabular}{|l|l}
\hline $\mathrm{N}(3)-\mathrm{S}(1)-\mathrm{C}(23)$ & $97.78(10)$ \\
\hline $\mathrm{O}(4)-\mathrm{S}(2)-\mathrm{O}(3)$ & $118.77(9)$ \\
\hline $\mathrm{O}(4)-\mathrm{S}(2)-\mathrm{N}(3)$ & $115.79(9)$ \\
\hline $\mathrm{O}(3)-\mathrm{S}(2)-\mathrm{N}(3)$ & $108.08(9)$ \\
\hline $\mathrm{O}(4)-\mathrm{S}(2)-\mathrm{C}(24)$ & $104.77(9)$ \\
\hline $\mathrm{O}(3)-\mathrm{S}(2)-\mathrm{C}(24)$ & $102.18(9)$ \\
\hline $\mathrm{N}(3)-\mathrm{S}(2)-\mathrm{C}(24)$ & $105.42(10)$ \\
\hline
\end{tabular}




\section{X-ray Crystallography Details of 16a}

Colorless needles of 16a were grown from a hexanes solution by slow evaporation at ambient temperature. A crystal of dimensions $0.37 \times 0.24 \times 0.24 \mathrm{~mm}$ was mounted on a Bruker SMART APEX CCD-based X-ray diffractometer equipped with a low temperature device and fine focus Mo-target X-ray tube $(\lambda=0.71073 \AA)$ operated at $1500 \mathrm{~W}$ power $(50 \mathrm{kV}, 30 \mathrm{~mA})$. The X-ray intensities were measured at $85(2) \mathrm{K}$; the detector was placed at a distance $5.055 \mathrm{~cm}$ from the crystal. A total of 2333 frames were collected with a scan width of $0.5^{\circ}$ in $\omega$ and $0.45^{\circ}$ in $\varphi$ with an exposure time of $25 \mathrm{~s} /$ frame. The frames were integrated with the Bruker SAINT software package with a narrow frame algorithm. The integration of the data yielded a total of 19089 reflections to a maximum $2 \theta$ value of $48.34^{\circ}$ of which 2510 were independent and 2007 were greater than $2 \sigma(\mathrm{I})$. The final cell constants (monoclinic, $\mathrm{a}=20.122(2) \AA ; \alpha=90^{\circ} ; \mathrm{b}=$ 7.1578(8) $\left.\AA ; \beta=115.372(6)^{\circ} ; \mathrm{c}=22.560(2) \AA ; \gamma=90^{\circ} ; \mathrm{V}=2935.9(5) \AA^{3}\right)$ were based on the xyz centroids of 7767 reflections above $10 \sigma(\mathrm{I})$. Linear absorption coefficient $\mu=$ $0.064 \mathrm{~mm}^{-1}$. Analysis of the data showed negligible decay during data collection; the data were processed with SADABS and corrected for absorption. The structure was solved and refined with the Bruker SHELXTL (version 2008/4) software package, using the space group $\mathrm{C} 2 / \mathrm{m}$ with $\mathrm{Z}=8$ for the formula $\mathrm{C}_{17} \mathrm{H}_{22} \mathrm{BN}\left(\mathrm{M}_{\mathrm{r}} 251.17 \mathrm{~g} / \mathrm{mol}\right.$, $\rho_{\text {calcd }}=$ $1.136 \mathrm{~g} / \mathrm{cm}^{3}$ ). All non-hydrogen atoms were refined anisotropically with the hydrogen atoms placed in idealized positions. There are two crystallographically independent molecules in the asymmetric unit, each lying on a mirror plane. Full matrix least-squares refinement based on $\mathrm{F}^{2}$ converged at $\mathrm{R} 1=0.0579$ and $\mathrm{wR} 2=0.1697$ [based on $\mathrm{I}>2 \sigma(\mathrm{I})$ ], $\mathrm{R} 1=0.0705$ and $\mathrm{wR} 2=0.1822$ for all data. CCDC-791460 contains the supplementary crystallographic data for 16a. The cif file can be obtained free of charge from Cambridge Crystallographic Data Center (http://www.ccdc.cam.ac.uk/products/csd/request/).

Sheldrick, G.M. SHELXTL, v. 2008/4, Bruker Analytical X-ray, Madison, WI, 2008; Sheldrick, G.M. SADABS, v. 2008/1, Program for Empirical Absorption Correction of Area Detector Data, University of Gottingen, Gottingen, Germany, 2008; Saint Plus, v. 7.60a, Bruker Analytical X-ray, Madison, WI, 2008. 


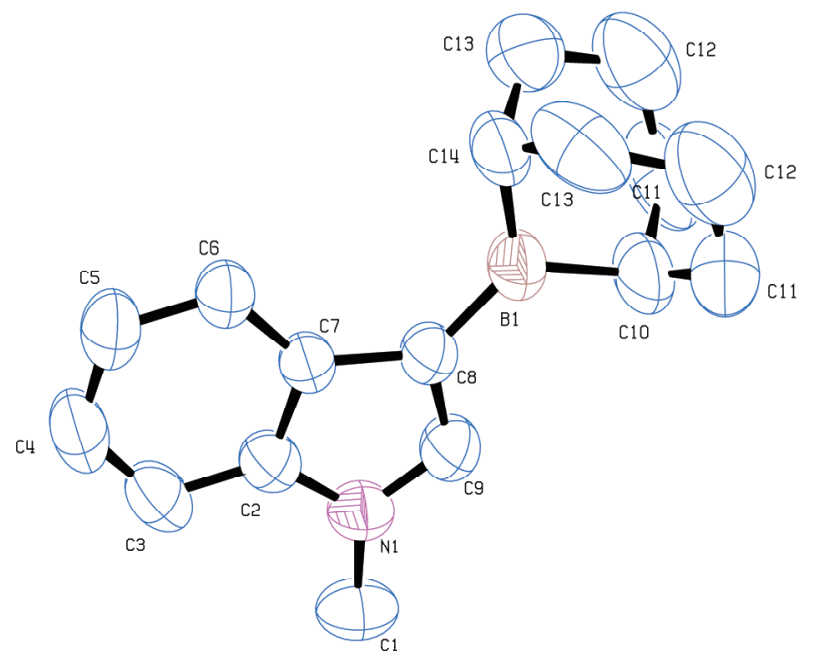

Bond lengths, Å:

\begin{tabular}{l|l|}
\hline $\mathrm{N}(1)-\mathrm{C}(9)$ & $1.345(4)$ \\
\hline $\mathrm{N}(1)-\mathrm{C}(2)$ & $1.388(4)$ \\
\hline $\mathrm{N}(1)-\mathrm{C}(1)$ & $1.460(3)$ \\
\hline $\mathrm{N}(2)-\mathrm{C}(23)$ & $1.343(4)$ \\
\hline $\mathrm{N}(2)-\mathrm{C}(16)$ & $1.386(3)$ \\
\hline $\mathrm{N}(2)-\mathrm{C}(15)$ & $1.462(3)$ \\
\hline $\mathrm{C}(2)-\mathrm{C}(3)$ & $1.391(4)$ \\
\hline $\mathrm{C}(2)-\mathrm{C}(7)$ & $1.401(4)$ \\
\hline $\mathrm{C}(3)-\mathrm{C}(4)$ & $1.366(5)$ \\
\hline $\mathrm{C}(4)-\mathrm{C}(5)$ & $1.393(5)$ \\
\hline $\mathrm{C}(5)-\mathrm{C}(6)$ & $1.378(4)$ \\
\hline $\mathrm{C}(6)-\mathrm{C}(7)$ & $1.403(4)$ \\
\hline $\mathrm{C}(7)-\mathrm{C}(8)$ & $1.467(4)$ \\
\hline $\mathrm{C}(8)-\mathrm{C}(9)$ & $1.384(4)$ \\
\hline
\end{tabular}

\begin{tabular}{|l|l|}
\hline $\mathrm{C}(8)-\mathrm{B}(1)$ & $1.516(4)$ \\
\hline $\mathrm{C}(10)-\mathrm{C}(11) \# 1$ & $1.539(4)$ \\
\hline $\mathrm{C}(10)-\mathrm{C}(11)$ & $1.539(4)$ \\
\hline $\mathrm{C}(10)-\mathrm{B}(1)$ & $1.583(5)$ \\
\hline $\mathrm{C}(11)-\mathrm{C}(12)$ & $1.501(4)$ \\
\hline $\mathrm{C}(12)-\mathrm{C}(13)$ & $1.499(4)$ \\
\hline $\mathrm{C}(13)-\mathrm{C}(14)$ & $1.539(3)$ \\
\hline $\mathrm{C}(14)-\mathrm{C}(13) \# 1$ & $1.539(3)$ \\
\hline $\mathrm{C}(14)-\mathrm{B}(1)$ & $1.566(5)$ \\
\hline $\mathrm{C}(16)-\mathrm{C}(17)$ & $1.391(4)$ \\
\hline $\mathrm{C}(16)-\mathrm{C}(21)$ & $1.414(4)$ \\
\hline $\mathrm{C}(17)-\mathrm{C}(18)$ & $1.375(4)$ \\
\hline $\mathrm{C}(18)-\mathrm{C}(19)$ & $1.376(4)$ \\
\hline $\mathrm{C}(19)-\mathrm{C}(20)$ & $1.390(4)$ \\
\hline
\end{tabular}

\begin{tabular}{|l|l}
\hline $\mathrm{C}(20)-\mathrm{C}(21)$ & $1.390(4)$ \\
\hline $\mathrm{C}(21)-\mathrm{C}(22)$ & $1.466(3)$ \\
\hline $\mathrm{C}(22)-\mathrm{C}(23)$ & $1.387(4)$ \\
\hline $\mathrm{C}(22)-\mathrm{B}(2)$ & $1.518(4)$ \\
\hline $\mathrm{C}(24)-\mathrm{C}(25) \# 2$ & $1.528(4)$ \\
\hline $\mathrm{C}(24)-\mathrm{C}(25)$ & $1.528(4)$ \\
\hline $\mathrm{C}(24)-\mathrm{B}(2)$ & $1.564(4)$ \\
\hline $\mathrm{C}(25)-\mathrm{C}(26)$ & $1.500(4)$ \\
\hline $\mathrm{C}(26)-\mathrm{C}(27)$ & $1.513(4)$ \\
\hline $\mathrm{C}(27)-\mathrm{C}(28)$ & $1.539(3)$ \\
\hline $\mathrm{C}(28)-\mathrm{C}(27) \# 2$ & $1.539(3)$ \\
\hline $\mathrm{C}(28)-\mathrm{B}(2)$ & $1.576(4)$ \\
\hline
\end{tabular}


Bond angles, deg.:

\begin{tabular}{|c|c|c|c|}
\hline $\mathrm{C}(9)-\mathrm{N}(1)-\mathrm{C}(2)$ & $107.9(2)$ & $\mathrm{N}(2)-\mathrm{C}(16)-\mathrm{C}(17)$ & $129.2(3)$ \\
\hline $\mathrm{C}(9)-\mathrm{N}(1)-\mathrm{C}(1)$ & $126.6(3)$ & $\mathrm{N}(2)-\mathrm{C}(16)-\mathrm{C}(21)$ & $108.1(2)$ \\
\hline $\mathrm{C}(2)-\mathrm{N}(1)-\mathrm{C}(1)$ & $125.5(2)$ & $C(17)-C(16)-C(21)$ & 122.7(3) \\
\hline $\mathrm{C}(23)-\mathrm{N}(2)-\mathrm{C}(16)$ & $107.8(2)$ & $\mathrm{C}(18)-\mathrm{C}(17)-\mathrm{C}(16)$ & $117.4(3)$ \\
\hline $\mathrm{C}(23)-\mathrm{N}(2)-\mathrm{C}(15)$ & $127.0(3)$ & $\mathrm{C}(17)-\mathrm{C}(18)-\mathrm{C}(19)$ & 121.3(3) \\
\hline $\mathrm{C}(16)-\mathrm{N}(2)-\mathrm{C}(15)$ & $125.2(3)$ & $\mathrm{C}(18)-\mathrm{C}(19)-\mathrm{C}(20)$ & $121.5(3)$ \\
\hline $\mathrm{N}(1)-\mathrm{C}(2)-\mathrm{C}(3)$ & 129.1(3) & $\mathrm{C}(21)-\mathrm{C}(20)-\mathrm{C}(19)$ & 119.3(3) \\
\hline $\mathrm{N}(1)-\mathrm{C}(2)-\mathrm{C}(7)$ & $108.0(2)$ & $C(20)-C(21)-C(16)$ & $117.9(2)$ \\
\hline $\mathrm{C}(3)-\mathrm{C}(2)-\mathrm{C}(7)$ & 122.9(3) & $C(20)-C(21)-C(22)$ & $134.8(2)$ \\
\hline $\mathrm{C}(4)-\mathrm{C}(3)-\mathrm{C}(2)$ & 117.7(3) & $C(16)-C(21)-C(22)$ & 107.3(2) \\
\hline$C(3)-C(4)-C(5)$ & $121.1(3)$ & $\mathrm{C}(23)-\mathrm{C}(22)-\mathrm{C}(21)$ & $103.2(2)$ \\
\hline $\mathrm{C}(6)-\mathrm{C}(5)-\mathrm{C}(4)$ & 121.2(3) & $\mathrm{C}(23)-\mathrm{C}(22)-\mathrm{B}(2)$ & 124.1(2) \\
\hline$C(5)-C(6)-C(7)$ & $119.3(3)$ & $\mathrm{C}(21)-\mathrm{C}(22)-\mathrm{B}(2)$ & $132.6(2)$ \\
\hline$C(2)-C(7)-C(6)$ & $117.8(2)$ & $\mathrm{N}(2)-\mathrm{C}(23)-\mathrm{C}(22)$ & $113.5(2)$ \\
\hline $\mathrm{C}(2)-\mathrm{C}(7)-\mathrm{C}(8)$ & $107.8(2)$ & $\mathrm{C}(25) \# 2-\mathrm{C}(24)-\mathrm{C}(25)$ & $114.0(3)$ \\
\hline$C(6)-C(7)-C(8)$ & $134.4(2)$ & $\mathrm{C}(25) \# 2-($ & $108.83(18)$ \\
\hline$C(9)-C(8)-C(7)$ & $103.2(2)$ & $\mathrm{C}(25)-\mathrm{C}(24)-\mathrm{B}(2)$ & $108.83(18)$ \\
\hline $\mathrm{C}(9)-\mathrm{C}(8)-\mathrm{B}(1)$ & 124.4(3) & $C(26)-C(25)-C(24)$ & $114.9(2)$ \\
\hline $\mathrm{C}(7)-\mathrm{C}(8)-\mathrm{B}(1)$ & $132.4(2)$ & $\mathrm{C}(25)-\mathrm{C}(26)-\mathrm{C}(27)$ & $114.0(2)$ \\
\hline $\mathrm{N}(1)-\mathrm{C}(9)-\mathrm{C}(8)$ & $113.2(3)$ & $C(26)-C(27)-C(28)$ & $115.2(2)$ \\
\hline $\mathrm{C}(11) \# 1-\mathrm{C}(10)-\mathrm{C}(11)$ & $113.4(3)$ & $\mathrm{C}(27)-\mathrm{C}(28)-\mathrm{C}(27) \# 2$ & $113.7(3)$ \\
\hline $\mathrm{C}(11) \# 1-\mathrm{C}(10)-\mathrm{B}(1)$ & $107.6(2)$ & $\mathrm{C}(27)-\mathrm{C}(28)-\mathrm{B}(2)$ & $107.84(17)$ \\
\hline $\mathrm{C}(11)-\mathrm{C}(10)-\mathrm{B}(1)$ & $107.6(2)$ & $\mathrm{C}(27) \# 2-\mathrm{C}(28)-\mathrm{B}(2)$ & $107.84(17)$ \\
\hline $\mathrm{C}(12)-\mathrm{C}(11)-\mathrm{C}(10)$ & $115.7(3)$ & $\mathrm{C}(8)-\mathrm{B}(1)-\mathrm{C}(14)$ & 127.3(3) \\
\hline $\mathrm{C}(13)-\mathrm{C}(12)-\mathrm{C}(11)$ & $114.3(2)$ & $\mathrm{C}(8)-\mathrm{B}(1)-\mathrm{C}(10)$ & $123.9(3)$ \\
\hline $\mathrm{C}(12)-\mathrm{C}(13)-\mathrm{C}(14)$ & $115.1(3)$ & $C(14)-B(1)-C(10)$ & $108.8(3)$ \\
\hline $\mathrm{C}(13) \# 1-\mathrm{C}(14)-\mathrm{C}(13)$ & $113.8(3)$ & $\mathrm{C}(22)-\mathrm{B}(2)-\mathrm{C}(24)$ & $126.7(2)$ \\
\hline $\mathrm{C}(13) \# 1-\mathrm{C}(14)-\mathrm{B}(1)$ & $108.26(19)$ & $C(22)-B(2)-C(28)$ & $124.9(3)$ \\
\hline $\mathrm{C}(13)-\mathrm{C}(14)-\mathrm{B}(1)$ & $108.26(19)$ & $\mathrm{C}(24)-\mathrm{B}(2)-\mathrm{C}(28)$ & $108.4(3)$ \\
\hline
\end{tabular}




\section{Computational Details}

All calculations were performed using Gaussian 09, Revision A.02 suite of computational programs. M. J. Frisch, G. W. Trucks, H. B. Schlegel, G. E. Scuseria, M. A. Robb, J. R. Cheeseman, G. Scalmani, V. Barone, B. Mennucci, G. A. Petersson, H. Nakatsuji, M. Caricato, X. Li, H. P. Hratchian, A. F. Izmaylov, J. Bloino, G. Zheng, J. L. Sonnenberg, M. Hada, M. Ehara, K. Toyota, R. Fukuda, J. Hasegawa, M. Ishida, T. Nakajima, Y. Honda, O. Kitao, H. Nakai, T. Vreven, J. A. Montgomery, Jr., J. E. Peralta, F. Ogliaro, M. Bearpark, J. J. Heyd, E. Brothers, K. N. Kudin, V. N. Staroverov, R. Kobayashi, J. Normand, K. Raghavachari, A. Rendell, J. C. Burant, S. S. Iyengar, J. Tomasi, M. Cossi, N. Rega, J. M. Millam, M. Klene, J. E. Knox, J. B. Cross, V. Bakken, C. Adamo, J. Jaramillo, R. Gomperts, R. E. Stratmann, O. Yazyev, A. J. Austin, R. Cammi, C. Pomelli, J. W. Ochterski, R. L. Martin, K. Morokuma, V. G. Zakrzewski, G. A. Voth, P. Salvador, J. J. Dannenberg, S. Dapprich, A. D. Daniels, O. Farkas, J. B. Foresman, J. V. Ortiz, J. Cioslowski, and D. J. Fox, Gaussian, Inc., Wallingford CT, 2009.

NBO analysis was performed using NBO Version 3.1 (E. D. Glendening, A. E. Reed, J. E. Carpenter, F. Weinhold), as implemented in Gaussian.

All calculations were performed in gas phase. Ultrafine integration grids (int=ultrafine) and very tight optimization criterion (opt=verytight) were used. The optimized structure was confirmed to be the local minimum by performing a frequency calculation. In addition, the wavefunction was tested for stability at the local minimum, confirming the closed-shell nature of the cation of $\mathbf{8 a}$. 


\title{
Cation of 8a
}

\section{M06-2X/6-31G(d,p):}

\author{
$E(M 06-2 X)=-991.365691904$
}

\begin{tabular}{|c|c|c|c|c|}
\hline 1 & B & -0.988854 & -0.154085 & 0.256678 \\
\hline 2 & $\mathrm{~N}$ & -0.033213 & 0.988445 & 1.125664 \\
\hline 3 & $\mathrm{~N}$ & 0.053602 & -1.546720 & 0.369601 \\
\hline 4 & $\mathrm{C}$ & 1.147787 & 1.254147 & 0.290508 \\
\hline 5 & $\mathrm{C}$ & 1.546464 & 2.509758 & -0.082125 \\
\hline 6 & $\mathrm{H}$ & 0.939664 & 3.380894 & 0.129490 \\
\hline 7 & $\mathrm{C}$ & 2.792394 & 2.694813 & -0.731627 \\
\hline 8 & $\mathrm{H}$ & 3.085645 & 3.694515 & -1.030252 \\
\hline 9 & $\mathrm{C}$ & 3.638453 & 1.637532 & -0.911660 \\
\hline 10 & $\mathrm{H}$ & 4.626753 & 1.783112 & -1.335508 \\
\hline 11 & $\mathrm{C}$ & 3.243723 & 0.323855 & -0.539811 \\
\hline 12 & $\mathrm{C}$ & 4.140024 & -0.768247 & -0.643619 \\
\hline 13 & $\mathrm{H}$ & 5.140822 & -0.589514 & -1.023379 \\
\hline 14 & $\mathrm{C}$ & 3.755065 & -2.019383 & -0.242524 \\
\hline 15 & $\mathrm{H}$ & 4.448677 & -2.851308 & -0.279789 \\
\hline 16 & $\mathrm{C}$ & 2.422069 & -2.253333 & 0.158851 \\
\hline 17 & $\mathrm{H}$ & 2.127563 & -3.274823 & 0.371864 \\
\hline 18 & $\mathrm{C}$ & 1.509163 & -1.226708 & 0.216832 \\
\hline 19 & $\mathrm{C}$ & 1.933659 & 0.110114 & -0.026706 \\
\hline 20 & $\mathrm{C}$ & -0.734129 & 2.248018 & 1.546753 \\
\hline 21 & $\mathrm{H}$ & 0.011916 & 2.931937 & 1.953509 \\
\hline 22 & $\mathrm{H}$ & -1.426851 & 1.983656 & 2.340168 \\
\hline 23 & $\mathrm{H}$ & -1.271942 & 2.721473 & 0.737397 \\
\hline 24 & $\mathrm{C}$ & 0.465433 & 0.483026 & 2.460704 \\
\hline 25 & $\mathrm{H}$ & 1.220864 & -0.284371 & 2.339102 \\
\hline 26 & $\mathrm{H}$ & -0.388269 & 0.110803 & 3.027646 \\
\hline 27 & $\mathrm{H}$ & 0.921581 & 1.319961 & 2.991396 \\
\hline 28 & $\mathrm{C}$ & -0.236194 & -2.452140 & -0.803680 \\
\hline 29 & $\mathrm{H}$ & 0.081741 & -1.954956 & -1.715405 \\
\hline 30 & $\mathrm{H}$ & -1.295991 & -2.677506 & -0.838277 \\
\hline 31 & $\mathrm{H}$ & 0.323427 & -3.378230 & -0.683765 \\
\hline 32 & $\mathrm{C}$ & -0.206941 & -2.391224 & 1.587989 \\
\hline 33 & $\mathrm{H}$ & 0.286542 & -3.356832 & 1.474657 \\
\hline 34 & $\mathrm{H}$ & -1.278911 & -2.550849 & 1.675225 \\
\hline 35 & $\mathrm{H}$ & 0.160522 & -1.917830 & 2.489928 \\
\hline 36 & $\mathrm{C}$ & -1.229562 & 0.265979 & -1.299409 \\
\hline 37 & $\mathrm{H}$ & -0.300517 & 0.288792 & -1.887581 \\
\hline 38 & $\mathrm{C}$ & -1.826456 & 1.686083 & -1.348722 \\
\hline 39 & $\mathrm{H}$ & -2.021171 & 1.948993 & -2.394169 \\
\hline 40 & $\mathrm{H}$ & -1.085817 & 2.420946 & -1.023156 \\
\hline 41 & $\mathrm{C}$ & -3.159048 & 1.792209 & -0.575503 \\
\hline 42 & $\mathrm{H}$ & -3.951223 & 1.496101 & -1.266659 \\
\hline 43 & $\mathrm{H}$ & -3.367692 & 2.844375 & -0.350208 \\
\hline 44 & $\mathrm{C}$ & -3.314961 & 0.966676 & 0.735336 \\
\hline 45 & $\mathrm{H}$ & -4.366390 & 0.664624 & 0.807440 \\
\hline 46 & $\mathrm{H}$ & -3.173341 & 1.624192 & 1.592518 \\
\hline 47 & $\mathrm{C}$ & -2.452068 & -0.329637 & 0.917457 \\
\hline 48 & $\mathrm{H}$ & -2.433166 & -0.545786 & 1.997036 \\
\hline 49 & $\mathrm{C}$ & -3.223737 & -1.479314 & 0.226178 \\
\hline 50 & $\mathrm{H}$ & -2.740756 & -2.449684 & 0.379644 \\
\hline 51 & $\mathrm{H}$ & -4.203032 & -1.581466 & 0.707339 \\
\hline 52 & $\mathrm{C}$ & -3.448578 & -1.210934 & -1.270657 \\
\hline 53 & $\mathrm{H}$ & -4.282115 & -0.509910 & -1.355466 \\
\hline 54 & $\mathrm{H}$ & -3.799959 & -2.128364 & -1.754370 \\
\hline 55 & $\mathrm{C}$ & -2.236849 & -0.660227 & -2.062227 \\
\hline 56 & $\mathrm{H}$ & -2.630531 & -0.081607 & -2.905958 \\
\hline 57 & $\mathrm{H}$ & -1.710776 & -1.489355 & -2.534675 \\
\hline
\end{tabular}


NMR Spectra 


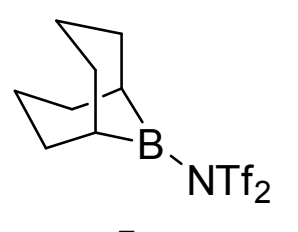

\section{$5 a$}

${ }^{1} \mathrm{H}$ NMR (400 MHz), $\mathrm{CD}_{2} \mathrm{Cl}_{2}$ 


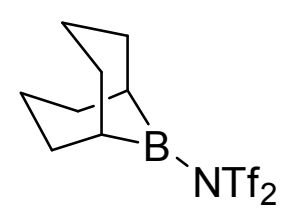

\section{$5 a$}

${ }^{11} \mathrm{~B}$ NMR (128 MHz), $\mathrm{CD}_{2} \mathrm{Cl}_{2}$

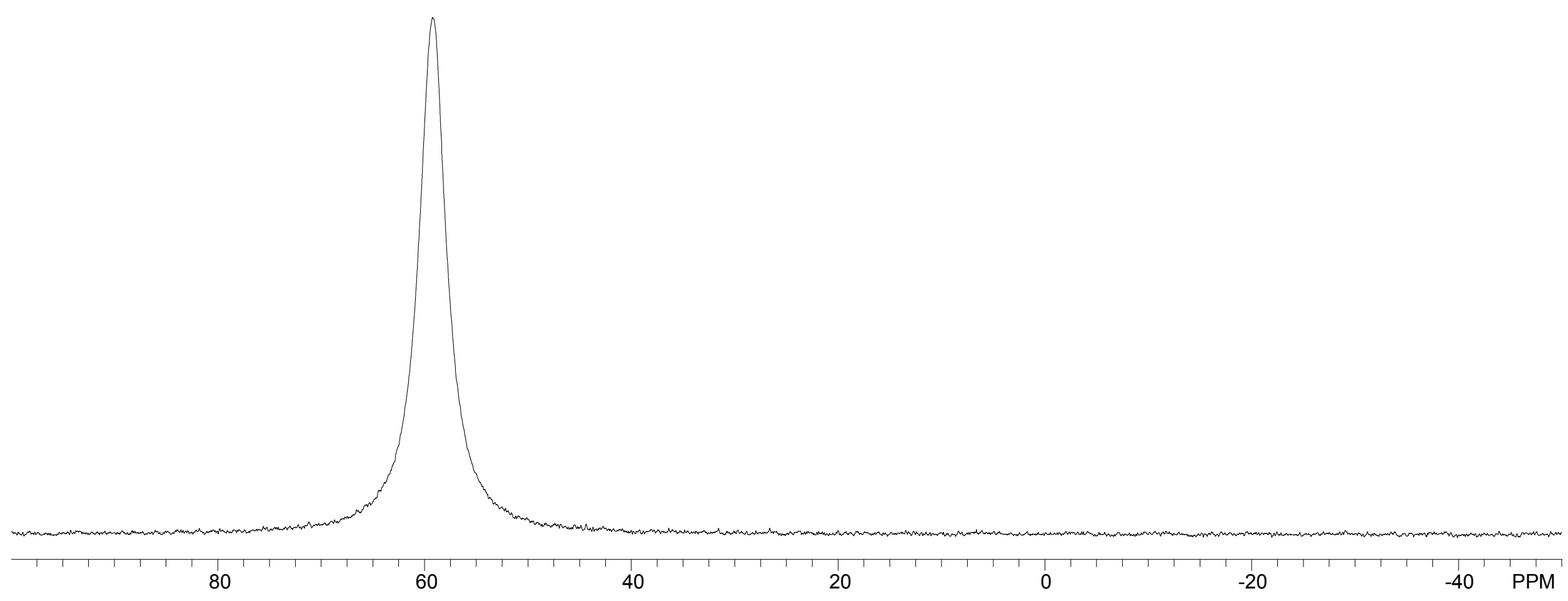




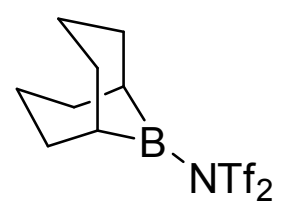

$5 a$

${ }^{13} \mathrm{C}\left\{{ }^{1} \mathrm{H}\right\}$ NMR (101 MHz), $\mathrm{CD}_{2} \mathrm{Cl}_{2}$ 


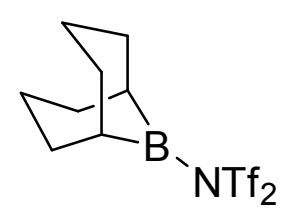

$5 a$

${ }^{19} \mathrm{~F}$ NMR (377 MHz), $\mathrm{CD}_{2} \mathrm{Cl}_{2}$ 


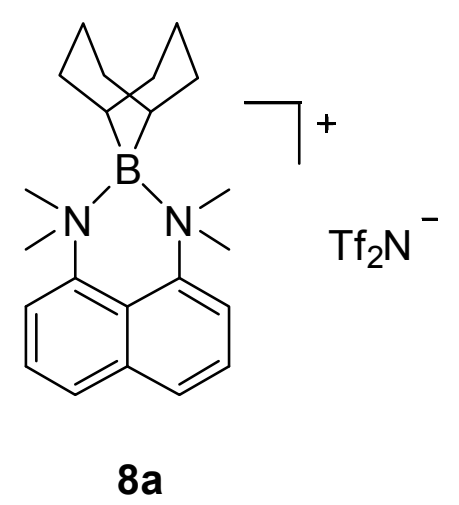

${ }^{1} \mathrm{H}$ NMR (500 MHz), $\mathrm{CD}_{2} \mathrm{Cl}_{2}$ 


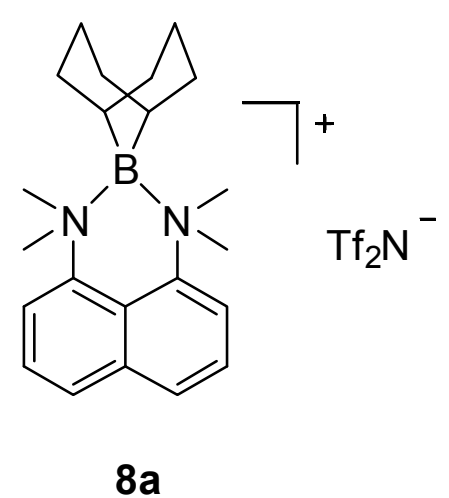

${ }^{11} \mathrm{~B}$ NMR (128 MHz), $\mathrm{CD}_{2} \mathrm{Cl}_{2}$ 


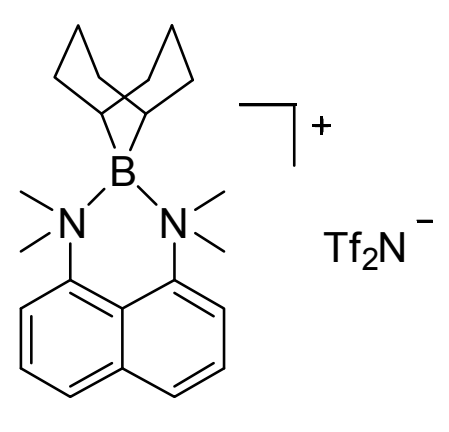

$8 a$

${ }^{13} \mathrm{C}\left\{{ }^{1} \mathrm{H}\right\}$ NMR (101 MHz),

$\mathrm{CD}_{2} \mathrm{Cl}_{2}$
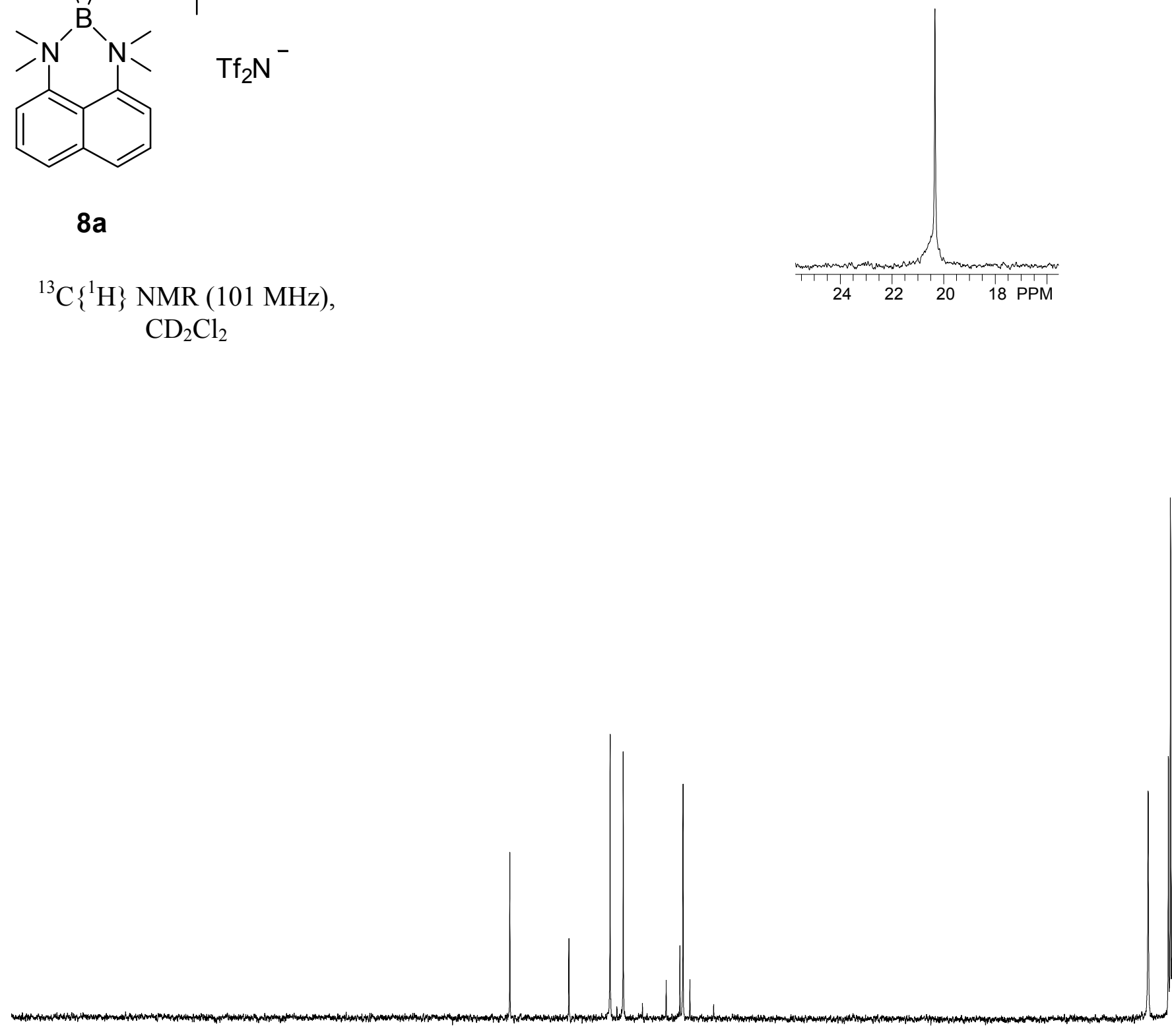


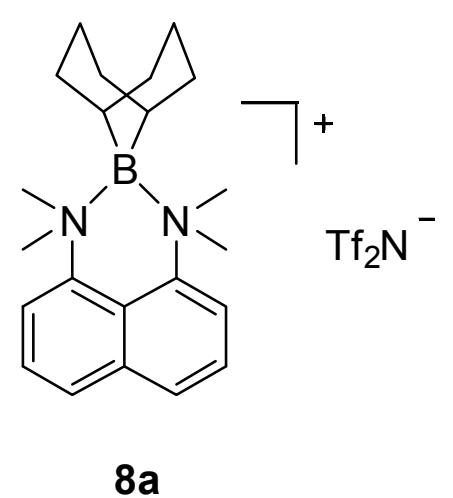

${ }^{19} \mathrm{~F}$ NMR (377 MHz), $\mathrm{CD}_{2} \mathrm{Cl}_{2}$ 


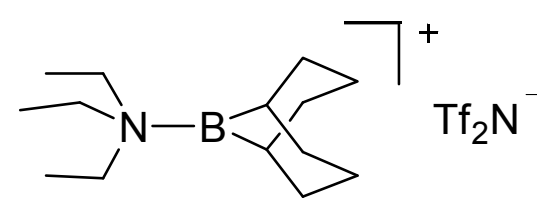

10

${ }^{1} \mathrm{H}$ NMR (400 MHz),

$\mathrm{CD}_{2} \mathrm{Cl}_{2}$ 


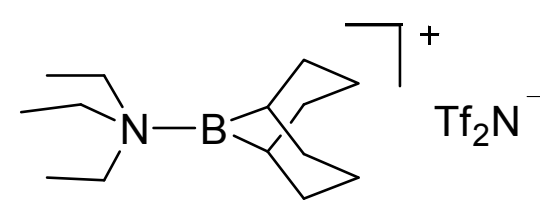

10

${ }^{11} \mathrm{~B}$ NMR (128 MHz), $\mathrm{CD}_{2} \mathrm{Cl}_{2}$

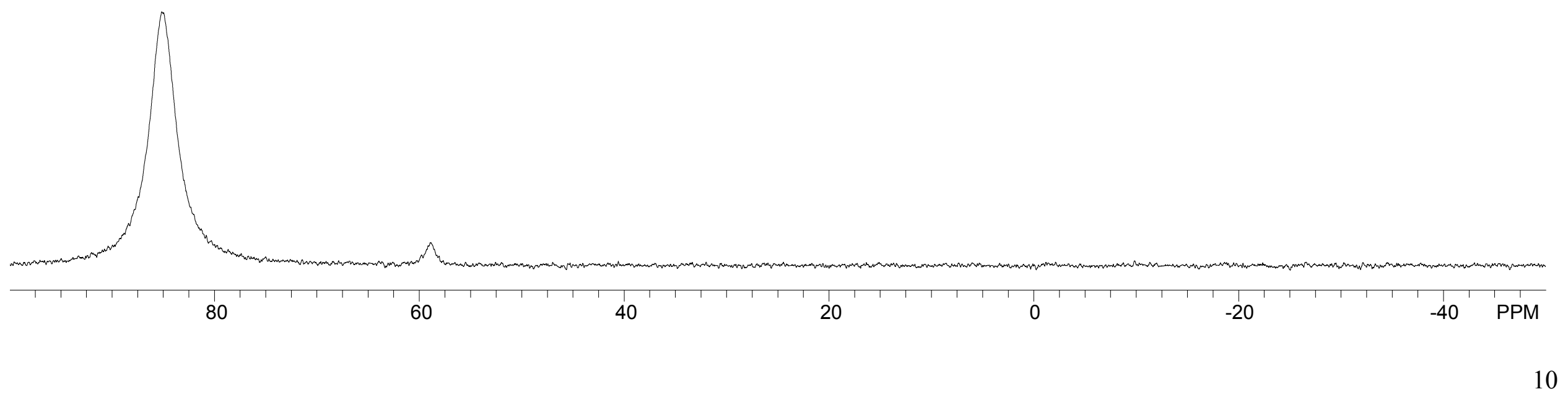




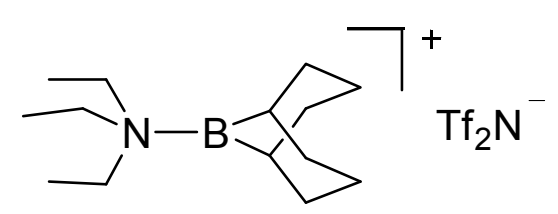

10

${ }^{13} \mathrm{C}\left\{{ }^{1} \mathrm{H}\right\}$ NMR (101 MHz), $\mathrm{CD}_{2} \mathrm{Cl}_{2}$ 


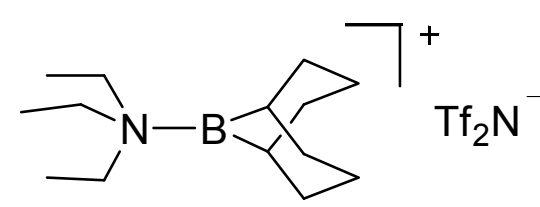

10

${ }^{19} \mathrm{~F}$ NMR (377 MHz), $\mathrm{CD}_{2} \mathrm{Cl}_{2}$ 


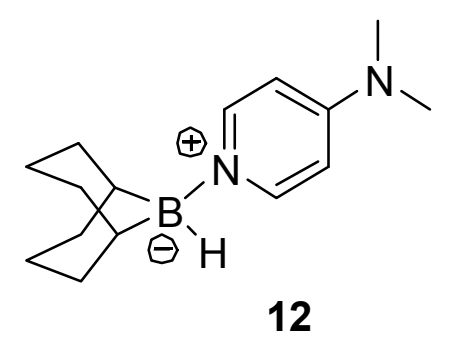

${ }^{1} \mathrm{H}$ NMR (500 MHz), $\mathrm{CD}_{2} \mathrm{Cl}_{2}$ 


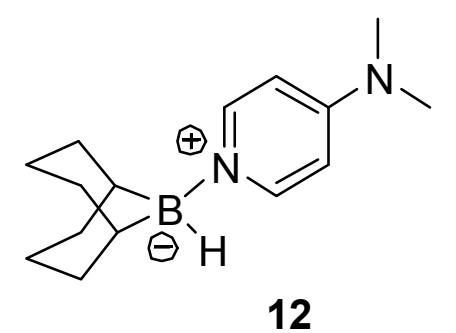

${ }^{11} \mathrm{~B}$ NMR (128 MHz), $\mathrm{CD}_{2} \mathrm{Cl}_{2}$ 


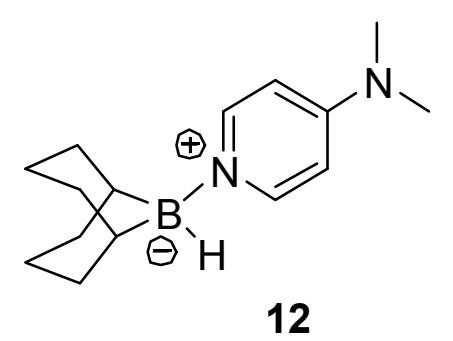

${ }^{11} \mathrm{~B}\left\{{ }^{1} \mathrm{H}\right\}$ NMR (128 MHz),

$\mathrm{CD}_{2} \mathrm{Cl}_{2}$ 


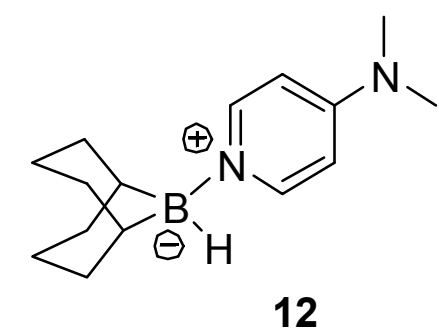

${ }^{13} \mathrm{C}\left\{{ }^{1} \mathrm{H}\right\}$ NMR (101 MHz), $\mathrm{CD}_{2} \mathrm{Cl}_{2}$

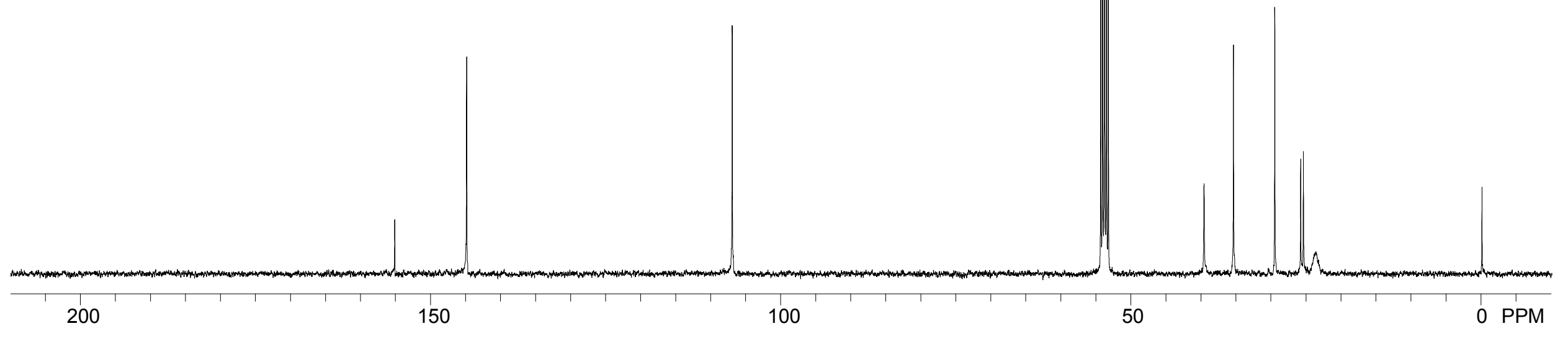




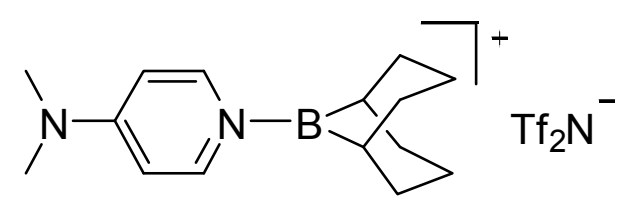

13

${ }^{1} \mathrm{H}$ NMR (500 MHz),

$\mathrm{CD}_{2} \mathrm{Cl}_{2}$

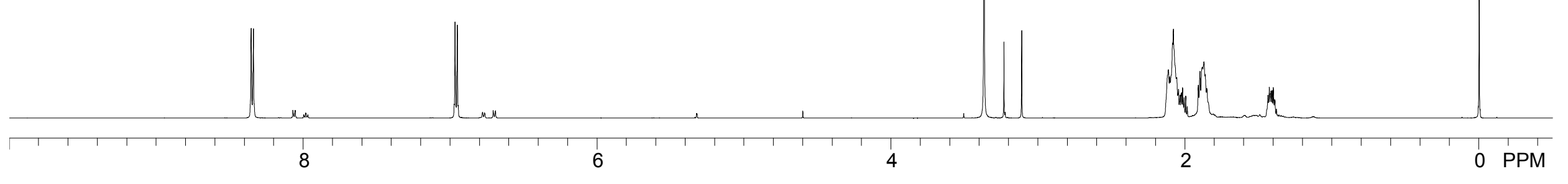




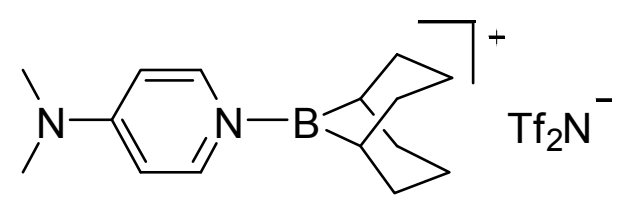

13

${ }^{11} \mathrm{~B}$ NMR (128 MHz), $\mathrm{CD}_{2} \mathrm{Cl}_{2}$

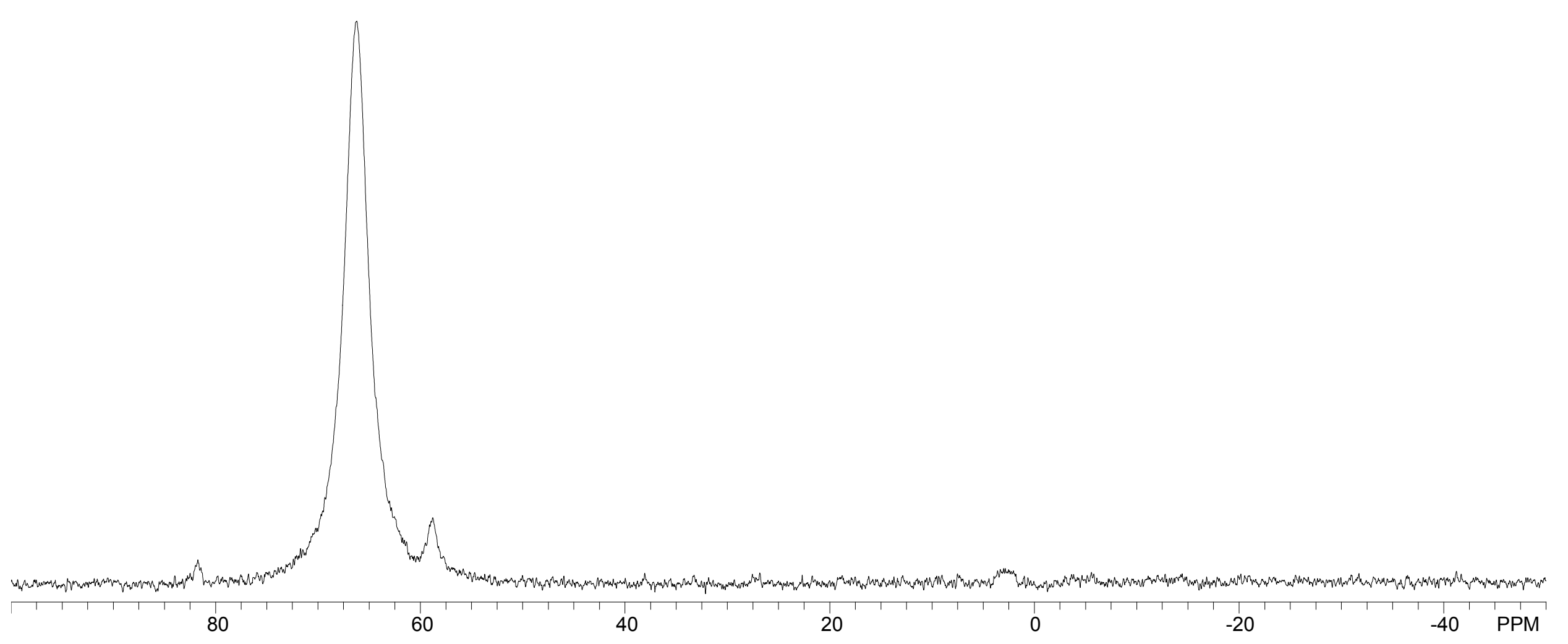




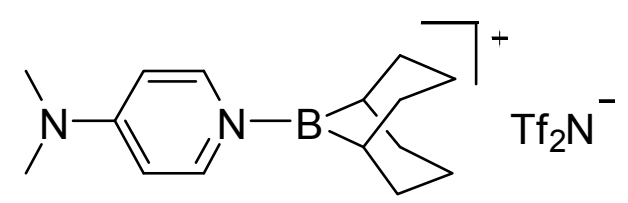

13

${ }^{13} \mathrm{C}\left\{{ }^{1} \mathrm{H}\right\}$ NMR (101 MHz), $\mathrm{CD}_{2} \mathrm{Cl}_{2}$ 


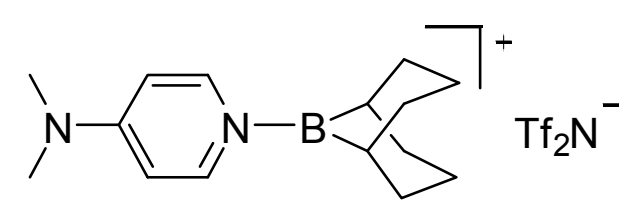

13

${ }^{19} \mathrm{~F}$ NMR (377 MHz), $\mathrm{CD}_{2} \mathrm{Cl}_{2}$ 


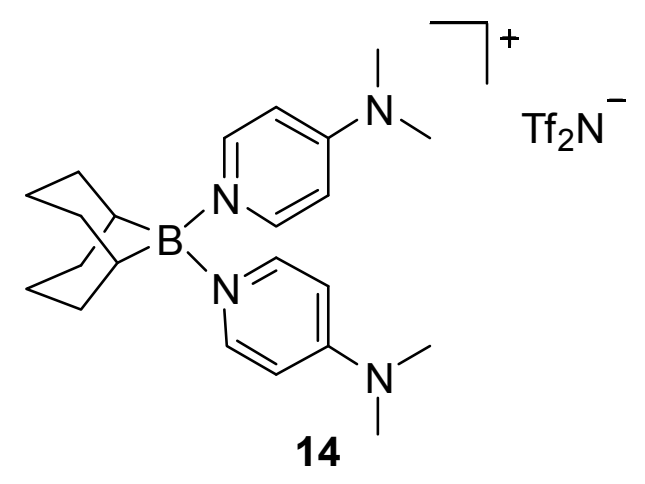

${ }^{1} \mathrm{H}$ NMR (500 MHz),

$\mathrm{CD}_{2} \mathrm{Cl}_{2}$

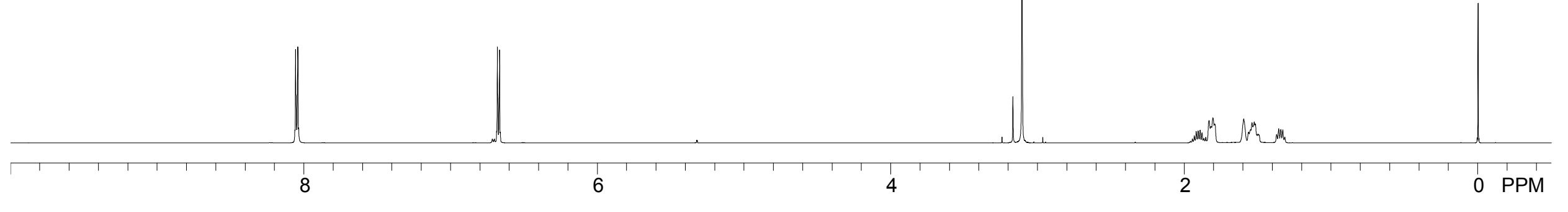




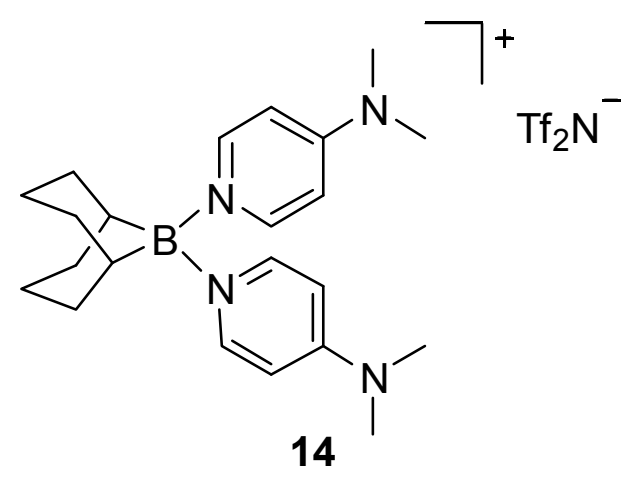

${ }^{11} \mathrm{~B}$ NMR (128 MHz), $\mathrm{CD}_{2} \mathrm{Cl}_{2}$

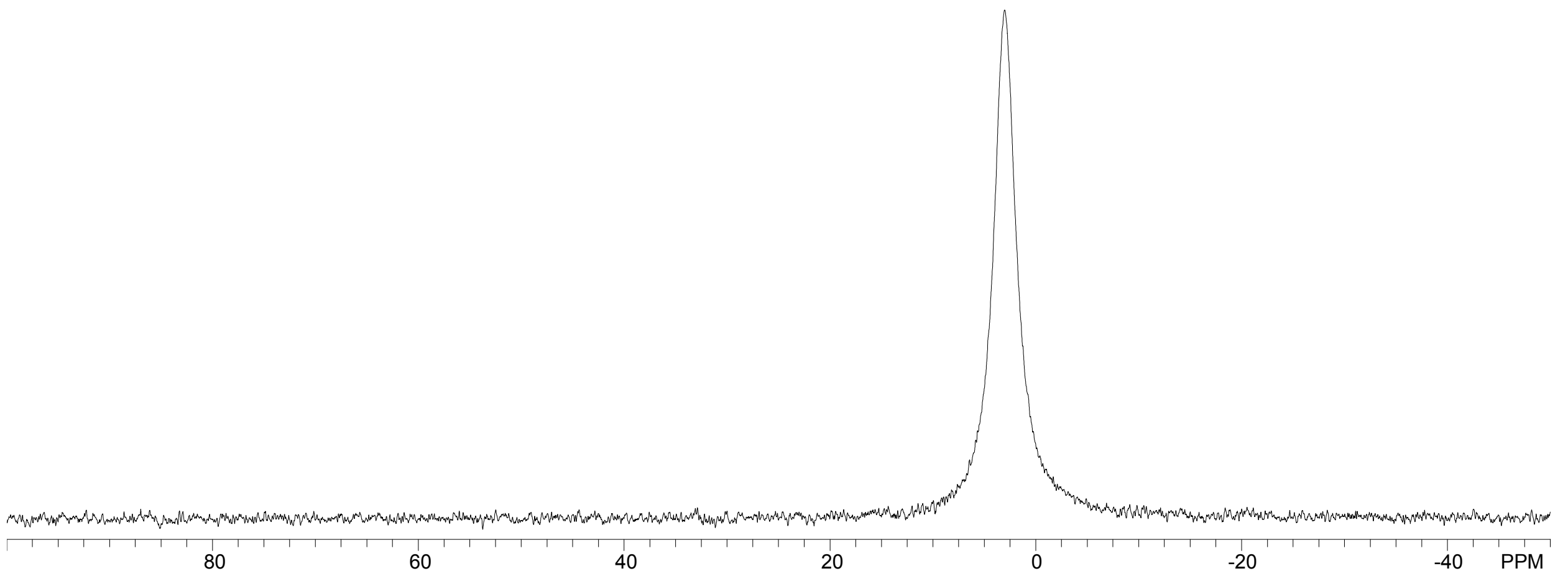




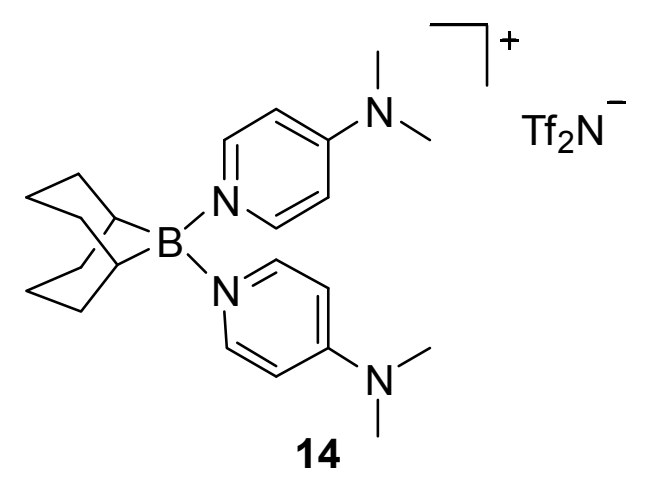

${ }^{13} \mathrm{C}\left\{{ }^{1} \mathrm{H}\right\}$ NMR (101 MHz), $\mathrm{CD}_{2} \mathrm{Cl}_{2}$

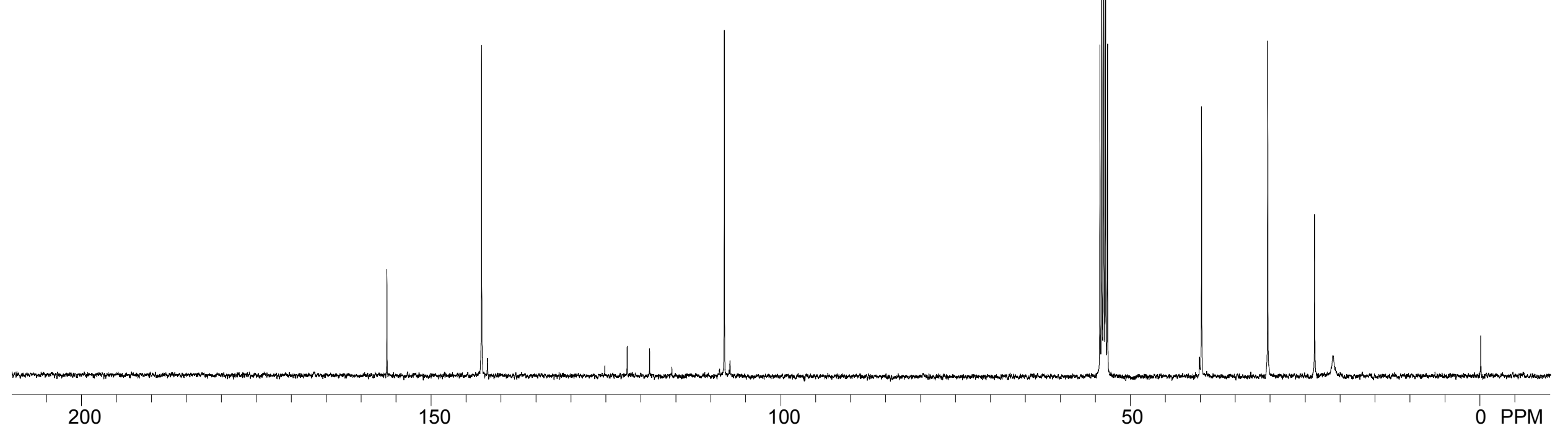




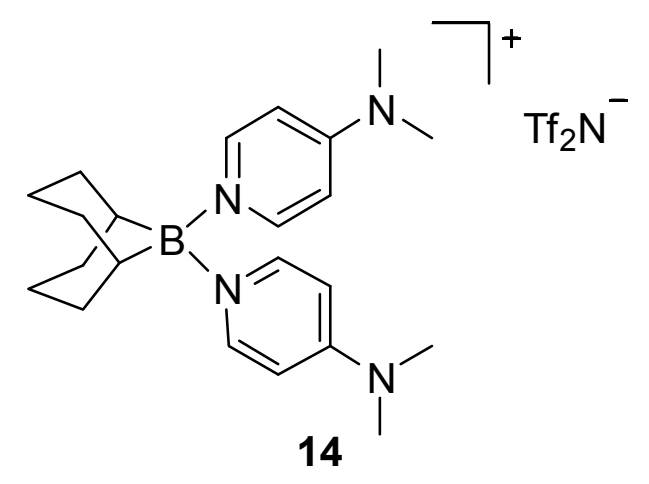

${ }^{19} \mathrm{~F}$ NMR (377 MHz), $\mathrm{CD}_{2} \mathrm{Cl}_{2}$ 


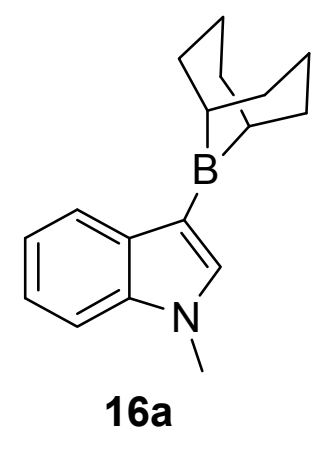

${ }^{1} \mathrm{H}$ NMR (500 MHz), $\mathrm{CD}_{2} \mathrm{Cl}_{2}$

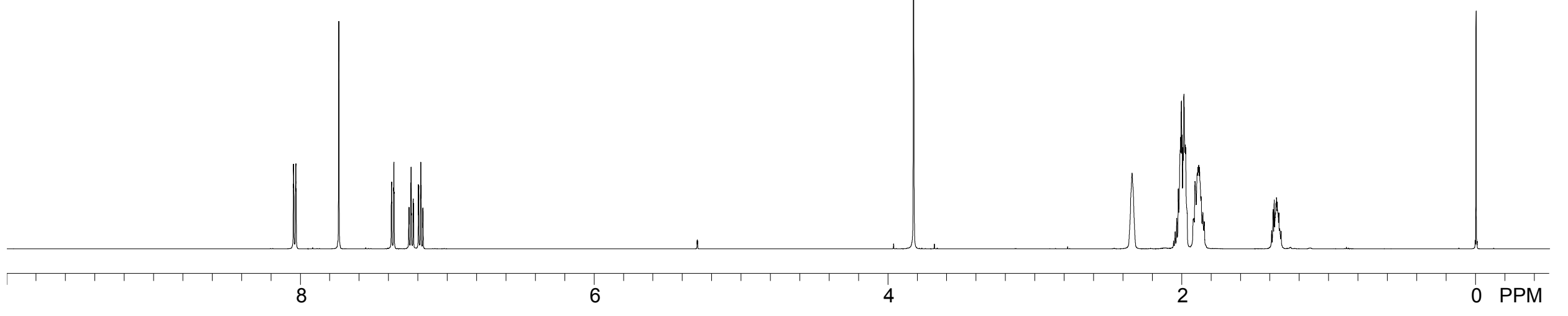




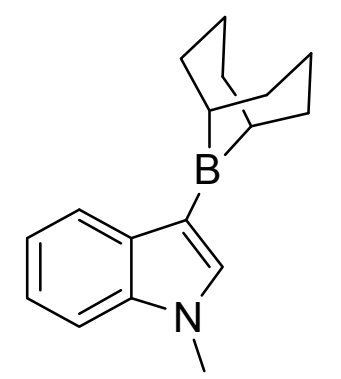

$16 a$

${ }^{11} \mathrm{~B}$ NMR (128 MHz), $\mathrm{CD}_{2} \mathrm{Cl}_{2}$

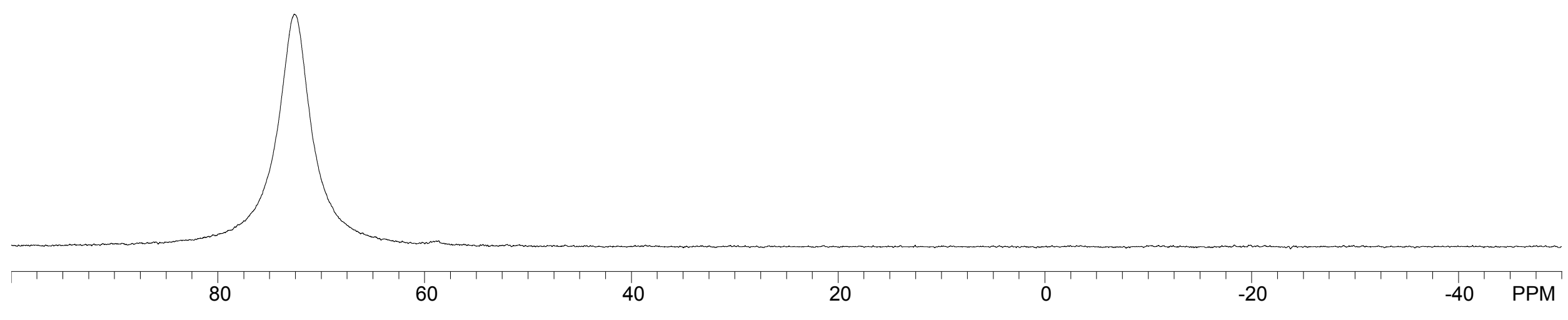



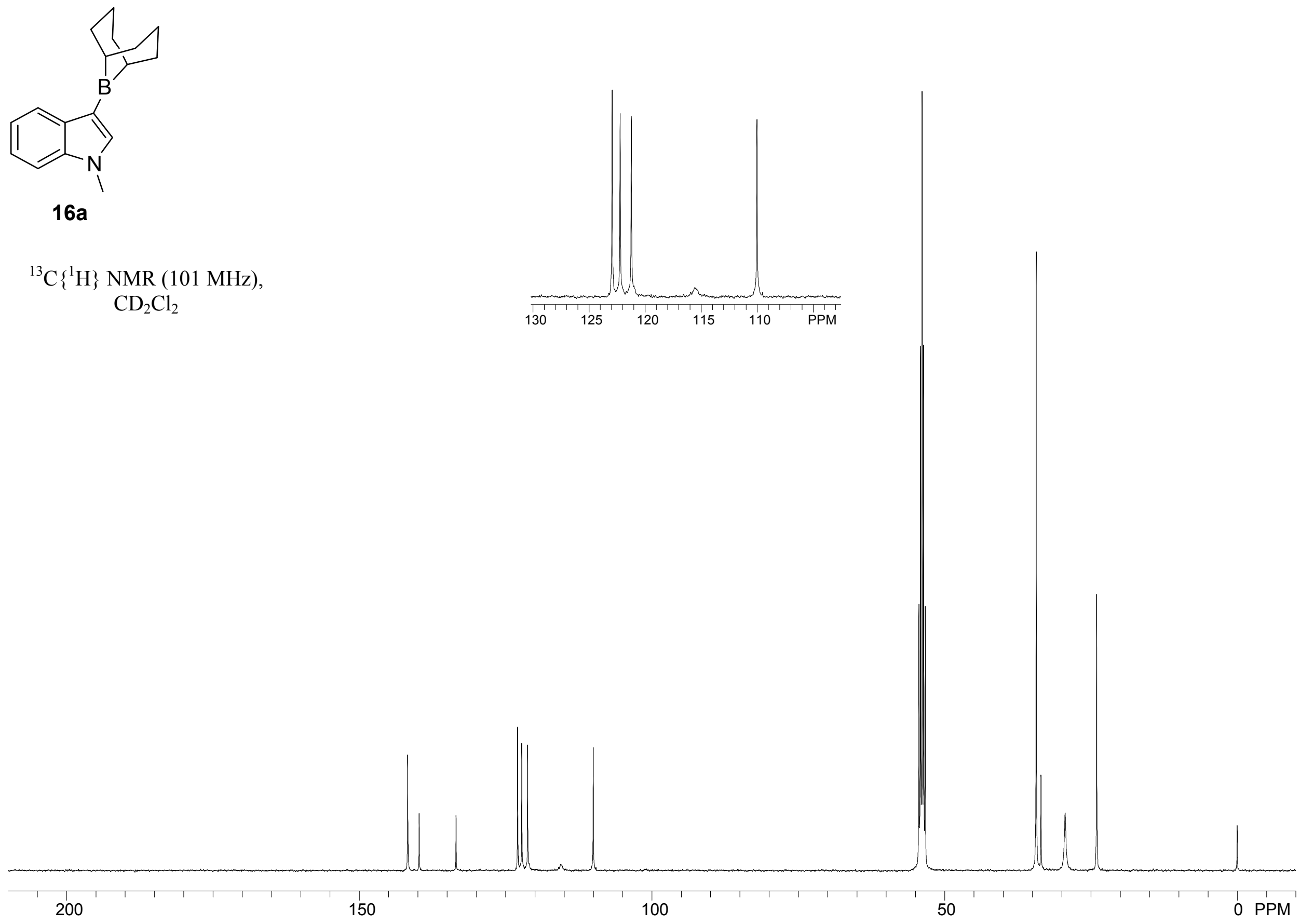


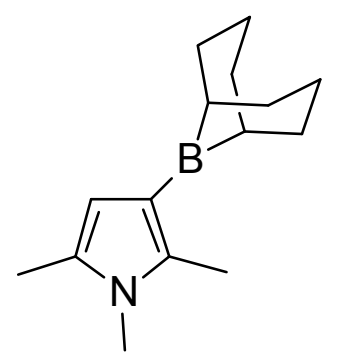

16b

${ }^{1} \mathrm{H}$ NMR (500 MHz), $\mathrm{CD}_{2} \mathrm{Cl}_{2}$ 


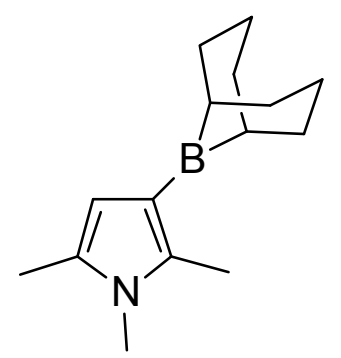

16b

${ }^{11} \mathrm{~B}$ NMR (128 MHz), $\mathrm{CD}_{2} \mathrm{Cl}_{2}$

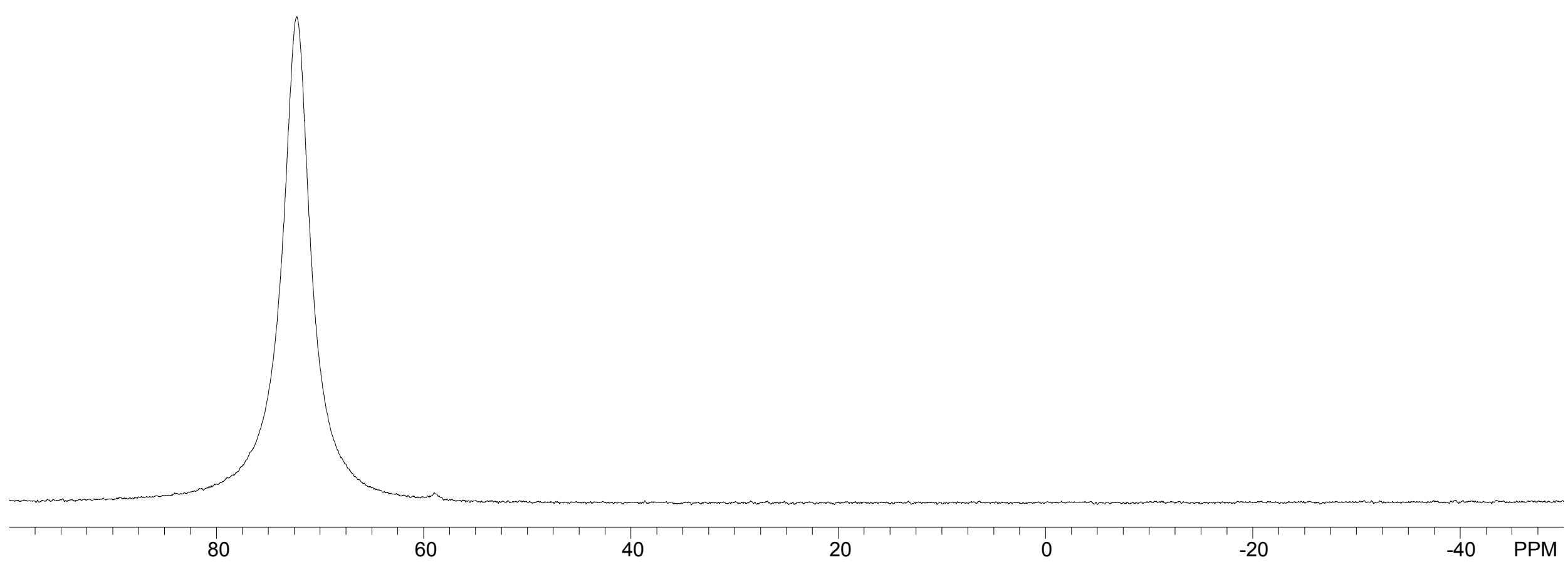




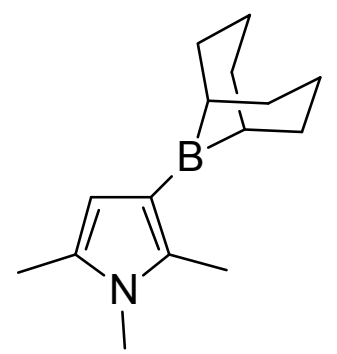

16b

${ }^{13} \mathrm{C}\left\{{ }^{1} \mathrm{H}\right\}$ NMR (101 MHz), $\mathrm{CD}_{2} \mathrm{Cl}_{2}$

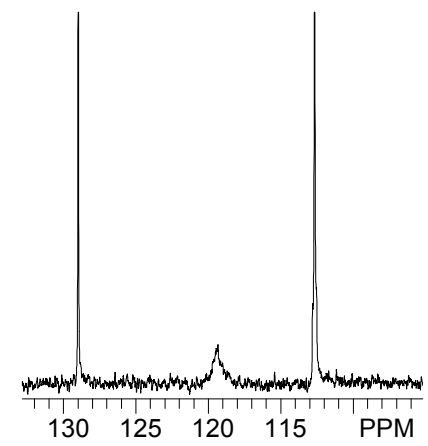




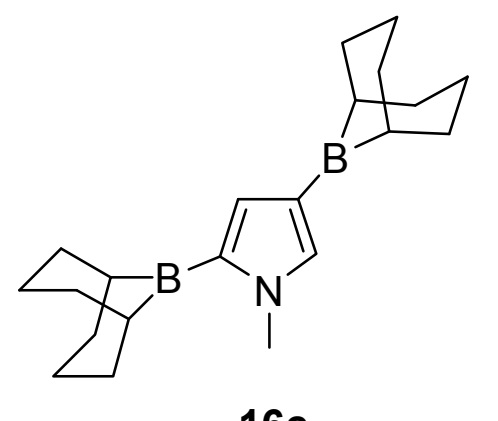

\section{$16 c$}

${ }^{1} \mathrm{H}$ NMR (500 MHz), $\mathrm{CD}_{2} \mathrm{Cl}_{2}$ 


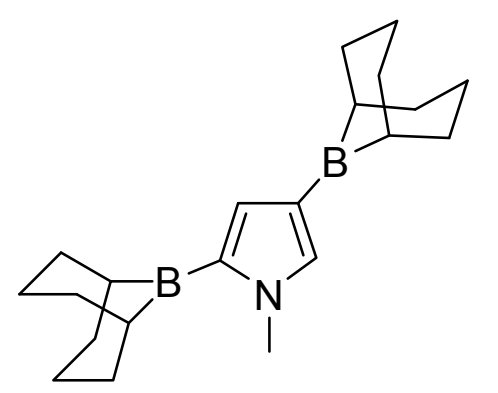

16c

${ }^{11} \mathrm{~B}$ NMR (128 MHz), $\mathrm{CD}_{2} \mathrm{Cl}_{2}$

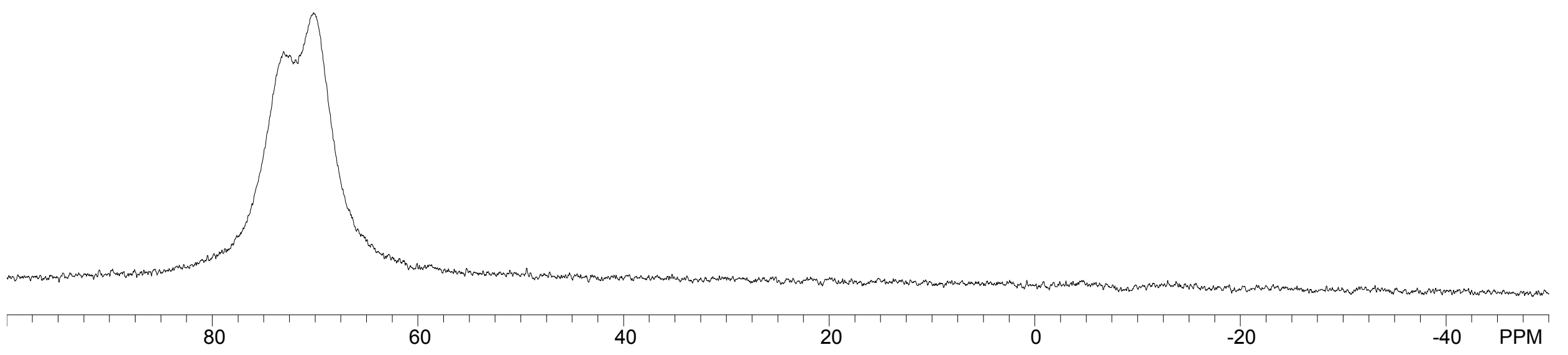




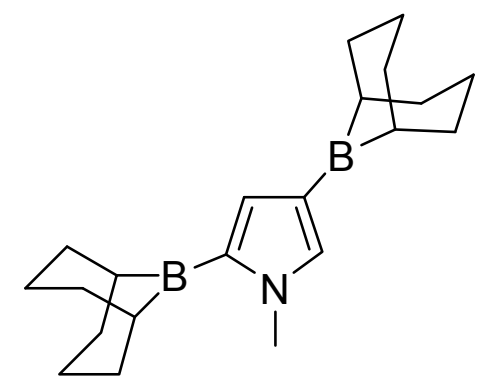

$16 c$

${ }^{13} \mathrm{C}\left\{{ }^{1} \mathrm{H}\right\}$ NMR (101 MHz), $\mathrm{CD}_{2} \mathrm{Cl}_{2}$

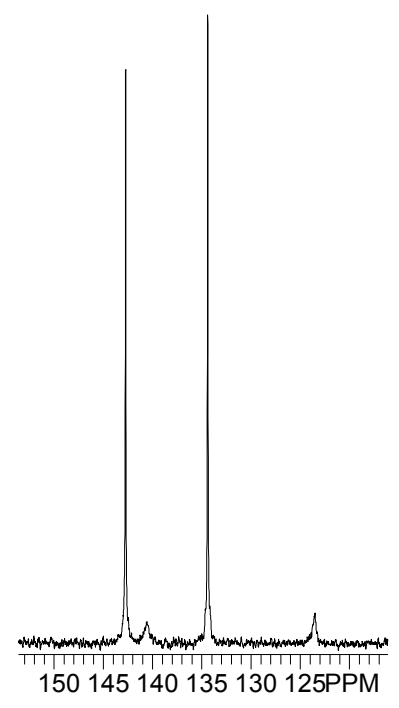




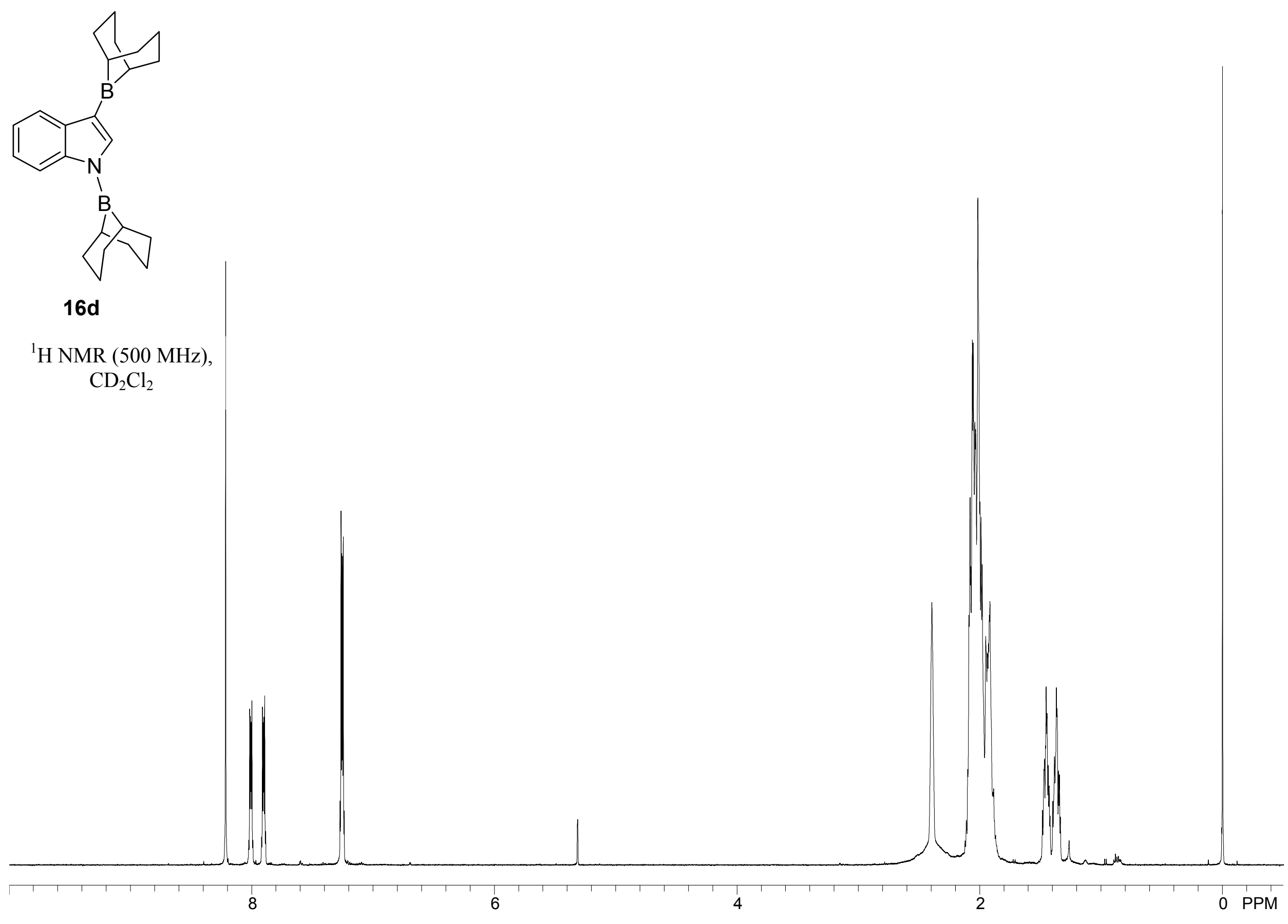




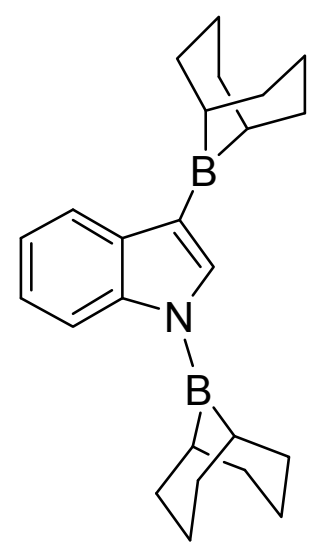

16d

${ }^{11}$ B NMR (128 MHz), $\mathrm{CD}_{2} \mathrm{Cl}_{2}$

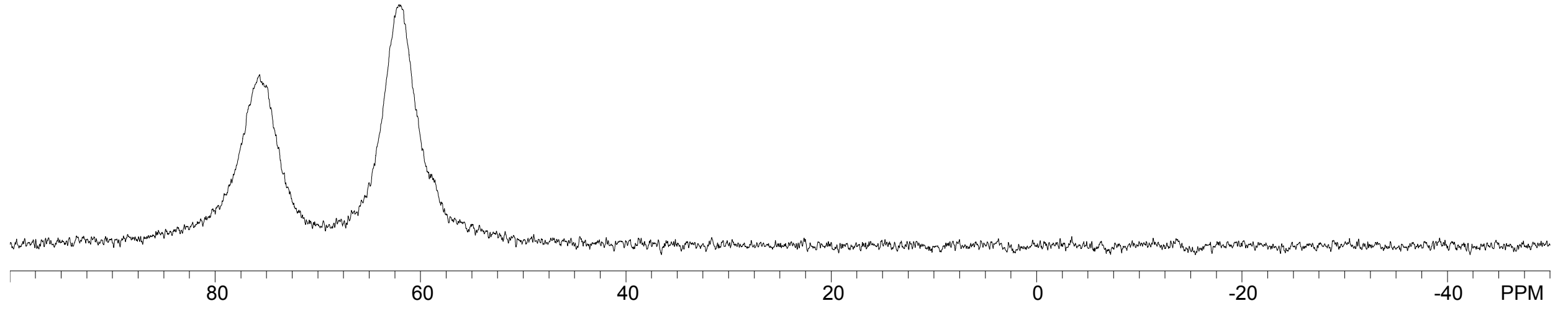




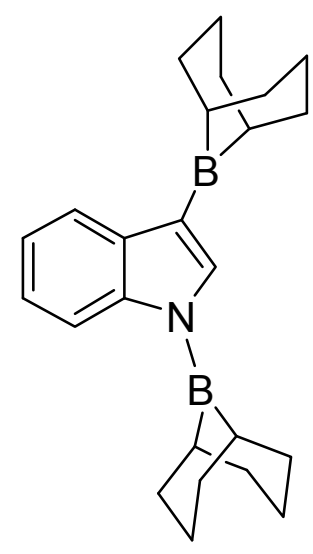

16d

${ }^{13} \mathrm{C}\left\{{ }^{1} \mathrm{H}\right\}$ NMR (101 MHz), $\mathrm{CD}_{2} \mathrm{Cl}_{2}$
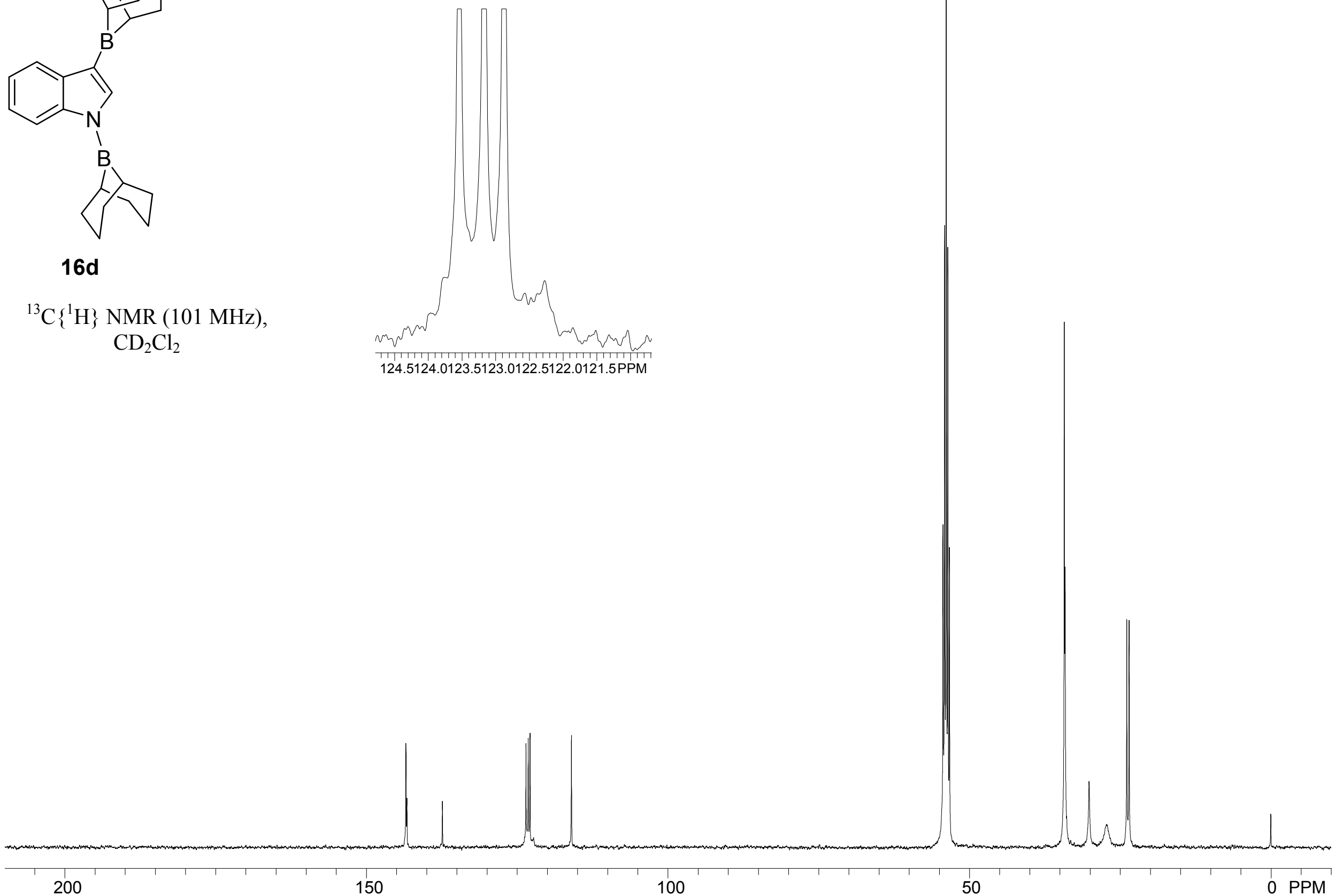